\title{
1.
}

\section{Ueber die Auflösung der transcendenten Gleichungen.}

\author{
(Vom Herrn Dr. M. A. Stern zu Göttingen.)
}

(Eine von der Königlich-Dänischen Gesellschaft der Wissenschaften gekrönte Preisschrift.)

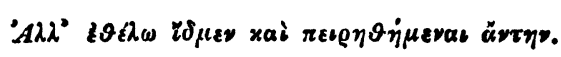

\section{Vorwort.}

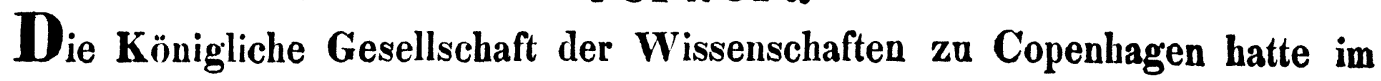
Jahre 1837 folgende Preisfrage gestellt.

Proponitur quaestio de aequationum transcendentium radicibus indagandis et quidem postulatur :

1. Ut plene et perfecte deducantur interque se comparentur methodi ipsarum radices inveniendi, ita ut quaenam cuiuscunque sint virtutes quaenam imperfectiones accurate indicetur, quibusve casibus unaquaeque sit magis minusve accommodata.

2. Ut diligenter inquiratur quatenus vel quibus saltem adhibitis cautionibus methodos, quibus vulgo in algebraicis aequationibus radices reales aut ab imaginariis separentur aut inter se, ad transcendentes quoque exteudere liceat.

3. Ut exponatur conspectus, quantum freri possit, plenus tam specialium aequationum quam generum earum, quae quidem forma transcendenti in gravissimis analyseos applicatae partibus occurrunt, simul cum regulis, quin fortasse tabulis ad usum ipsum accomodatis, quibus revera faciliores ac breviores reddantur calculi illi radicum, alias saepe prolixissimi.

Das Folgende ist ein genauer Abdruck der Schrift, die ich der Königl. G. d. W. in December 1837 überreicht habe; ich habe mir nur einige aufserwesentliche Aenderungen erlaubt, wie namentlich, dafs ich da, wo ich, der Bestimmung der Schrift gemäls, von meinen eigenen Arbeiten als denen eines Dritteu sprechen mufste, dies nun geändert habe.

Göttingen, den 5. Januar 1840. 
Die Konigliche Societät der Wissenschaften hat als Preisfrage die Aufgabe gestellt, die Wurzeln der transẹendenten Gleichungen zu finden. Indem ich es unteruehme, diese Frage zu beantworten, will ich zuvor Folgendes bemerken. Ich setzte voraus, dafs die Königl. Societät d. W. nur die Auflösung der numerischen transcendenten Gleichungen verlangt, und zwar blors solcher, in welchen nur eine unbekannte Grölse vorkommt. Eine allgemeine Auflösung der litteralen transcendenten Gleichungen kann wohl nach dem gegenwärtigen Zustande der Wissenschaft nicht verlangt werden, da man noch nicht im Stande ist, dasselbe in Beziehung auf die litteralen algebraischen Gleichungen zu leisten. Aus demselken Grunde glaube ich, die Aufgabe, aus mehreren transcendenten Gleichungen, in welchen eben so viele unbekannte Gröfsen vorkommen, die Werthe dieser Gröfsen zu finden, bei Seite setzen zu dürfen. Um diese Aufgabe zu lösen, mürste man alle unbekannten Grölsen, bis auf eine, eliminiren. Diese Elimination aber, welche schon in dem Falle, wenn die Gleichungen algebraische sind, bedeutende Schwierigkeiten darbietet, störst bei den transcendenten Gleichungen auf unübersteigliche Hindernisse. Ich werde daher die Untersuchung darauf beschränken, zu zeigen, wie man bei jeder gegebenen numerischen transcendenten Gleichung die reellen Wurzeln mit beliebiger Genauigkeit berechnen und das Vorhandensein der imaginären Wurzeln entdecken kann. Was dagegen die numerischen Werhe der imaginären Wurzeln betrifft, so kann deren Berechnung um so weniger verlangt werden, da bis jetzt keine Methode existirt, die solches mit Bequemlichkeit für die algebraischen Gleichungen leistet. Die einzige sichere Methode, welche man besitzt, um die Werthe der imaginären Wurzeln algebraischer Gleichungen zu finden, ist die, welche Fourier zuerst angedeutet hat. Ich werde später zu zeigen suchen, in wiefern diese Methode auf die transcendenten Gleichungen anwendbar ist.

Die Societăt d. W. hat in dem Programme die Frage in drei Theile getheilt. Ich werde, dieser Eintheilung folgeud, zuerst von den vorhandenen Methoden zur Auflösung der transcendenteu Gleichungen sprechen; alsdanu zeigen, wie man diese Gleichungen wirklich auflösen kann, und zuletzt Anwendungen davon auf besonders häufig vorkommende Beispiele machen. 


\section{Aeltere Methoden.}

1. Eine besondere Behandlung der Auflösung der transcendenten Gleichungen fundet sich, so viel mir bekannt ist, nirgendwo. In einzelnen Fällen hat man solche Gleichungen entweder durch blofses Probiren aufzulösen gesucht, welches Verfabren, als unsicher und unwissenschaftlich, keine weitere Beachtung verdient, oder man hat die bereits bekannten Methoden zur Auflösung der algebraischen Gleichungen auch auf die transcendenten ausgedehnt. Bekanntlich hat aber zuerst Lagrange eine sichero Methode zur Auflösung der algebraischen Gleichungen gegeben, während die älteren Methoden nit Mängeln behaftet sind, die sie ganz unbrauchbar machen und die ich hier nicht besonders hervorzuheben brauche, da dieser grofse Mathematiker sie bereits in das hellste Licht gesetzt hat. Es versteht sich daher von selbst, dafs die Anwendung dieser älteren Methoden auf die transcendenten Gleichungen dieselben Mängeln haben; wo sie häufig noch viel bedeutender sein können.

So z. B. wendet Euler ${ }^{*}$ ) die Newtonsche Approximations-Methode an, um mehrere transcendente Gleichungen aufzulösen. Die Mängel dieser Methode hat aber bereits Lagrange ${ }^{*}$ *) nachgewiesen. An einem andern Orte ${ }^{*}$ hat Euler die Bernoullische Methode angewandt um die kleinste Wurzel der Gleichung

$$
\frac{1}{2}=z-\frac{z^{3}}{2.3}+\frac{z^{5}}{2.3 .4 .5}-\frac{z^{7}}{2.3 .4 .5 .6 .7}+\ldots
$$

zu finden, wobei er jedoch selbst bemerkt, dals diese Methode nur selten zur Erfindung der Wurzeln transcendenter Gleichungen angewandt werden könne. Dieselbe Methode bat Euler auch später benutzt $\dagger^{\dagger}$ ), um die kleinsten Wurzeln einiger transcendenten Gleichungen zu finden. Die ausführliche Darstellung, welche Euler $+\dagger$ ) der Bernoullischen Methode gewidmet hat und die Bemerkungen, welche Lagrange später hinzugefügt hat, zeigen zar Genüge, dafs die Anwendung dieser Methode, selbst auf algebraische Gleichungen, sehr beschränkt ist und daher noch weniger zur allgemeinen Aaflösung der transcendenten Gleichungen gebraucht werden kann. Man er-

-) Instit. calc. diff. T. II. $\$ .242$ seqq.

*) Résol. des équat. num. No. V.

**) Introd. in anal. inf. 1. I. \$ 355.

†) Nova act. Acad. Petr. Tom. IX. p. 28 seqq.

if) Introd. in an. inf. 1. I. C. 17. 
hält nemlich vermittelst dieser Methode nur Näherungswerthe der gröfsten und kleinsten Wurzel, und zwar nur in dem Falle, wenn die Gleichung kein Paar zusammengehörender imaginärer Wurzeln hat, deren Product grïfser ist als das Quadrat der gröfsten reellen Wurzel. Im entgegengesetzien Falle ist die Methode unbrauchbar. In der neuesten Zeit hat freilich Fourier *) Andeutungen gegeben, wie die Bernoullische Methode verbessert und zur Auffindung aller Wurzeln gebraucht werden könne und ich **) habe nachgewiesen, dafs sich diese Andeutungen allerdings realisiren lassen. In wie fern nun diese Ausbildung der Bernoullischen Methode sich auch auf die transceudenten Gleichungen anwenden lasse, werde ich später untersuchen.

Die Lagrange'sche Methode zur Auflösung der algebraischen Gleichungen ist zwar, von theoretischer Seite betrachtet, streng richtig, aber in ihrer Anwendung, wie bekannt, grofsen Schwierigkeiten unterworfen. Ihr wesentlichster Mangel liegt darin, dafs man eine Zahl kennen mufs, die kleiner als der kleinste Unterschied der Wurzeln ist. Die Aufsuchung dieser Zahl aber erfordert, sobald die Gleichung von einem hohen Grade ist, fast unausführbare Rechnungen; wie es Lagrange selbst schon bemerkt hat. Noch viel schwieriger mufs die Aufsuchung dieser Zahl sein, sobald die gegebene Gleichung eine transcendente ist, und man kann sie in diesem Falle, wie auch schon Poison bemerkt hat ***;*), als völlig unausführbar ansehen.

2. In dem 24sten Bande der Memoiren der Berliner Academie hat Lagrange eine Methode gegeben, um die Wurzeln der algebraischen Gleichungen durch unendliche Reihen auszudrücken, und es haben später mehrere bedeutende Mathematiker diese Untersuchung aufgenommen und vervollkommnet. Diese Methode kommt zuletzt auf die Umkehrung der Reihen zurück, indem sie zeigt wie man, wenn eine Gleichung

$$
y=a+b x+c x^{2}+d x^{3}+e x^{4} \ldots
$$

gegeben ist, den Werth von $x$ durch eine nach Potenzen von $y$ georduete Reihe ausdrücken kann, so dafs man

$$
x=\boldsymbol{A}+\boldsymbol{B} y+\boldsymbol{C} y^{2}+\ldots
$$

hat. Lagrange bemerkt im Eingange zu dieser Abhandlung, dafs diese

-) Analyse des équations p. 6. 8 seqq.

* Crelle Journ. für die Math. Bd. 11. p. 293 ff.

*) Journ. de l'école polyt. cah. 19. p. 382. 
Methode auch bei den transcendenten Gleichungen brauchbar sei, welche Logarithmen und Kreisbogen enthalten, woraus also hervorgeht, dafs er sie selbst nicht für eine allgemein auf transcendente Gleichungen anwendbare Methode gehalten hat. In der That ist sie aber auch erheblichen Schwierigkeiten unterworfen. Soll nemlich der Werth von $x$ durch die nach Potenzen von $\gamma$ geordnete Reibe gefunden werden, so mufs diese Reihe convergiren; sie kann aber auch häufig divergiren und die Untersuchung, ob das eine oder das andere Statt findet, ist schwierig, da die Reihen häufig sehr verwickelt sein können. Lagrange selbst hat zwar gesucht Kennzeichen anzugeben, vermittelst welcher man die Convergenz oder Divergenz beurtheilen könne : diese Kennzeichen sind aber schon aus dem Grunde ungenügend, weil sie auf einer falschen Definition der Convergenz beruhen. Lagrange nennt nemlich convergirend eine Reihe, deren Glieder unendlich klein werden, und er sucht daher nur zu bestimmen, ob die Reihen, welche die Wurzeln der Gleichungen ausdrücken, diese Eigenschaft baben, oder nicht. Es ist aber hiulänglich bekannt, dafs die Glieder einer Reihe unendlich klein werden können, während die Reihe dennoch divergirt, das heifst, während ihr Werth über alle Grenzen hinaus wächst und also zur Berechnung untauglich ist. Hierzu kommt noch, dafs wenn man auch den Werth einer Wurzel durch eine convergirende Reihe gefunden hat, es doch äufserst schwierig ist, die verschiedenen Reihen, welche verschiedene Wurzeln ausdrücken, zu finden und von einander zu unterscheiden; wie man es schon aus Lagrange's Untersuchungen sehen kanu, die sich nur auf algebraische Gleichungen beziehen. Auch darf nicht übersehen werden, dafs es nicht möglich ist, auf diesem Wege die einzelnen Wurzeln allmälig nach ihrer Grölse zu finden; was doch ein wesentliches Erfordernifs einer tauglichen Auflösungsmethode ist, da es besonders bei den transcendenten Gleichungen in der Regel nur darauf ankommt, die kleinsten Wurzeln zu kennen. In dem besonderen Falle, wo man den Werth der Wurzeln schon beinahe kennt, kann diese Methode allerdings of mit Nutzen gebraucht werden, um genauere Werthe zu finden.

3. Einen anderen Weg hat Cauchy eingeschlagen *). Seine Methode' beruht auf dem Verfahren, dessen sich Legendre bedient * ${ }^{*}$ ), um einen

*) Leçons sur le calcul différentiel ch. 14.

* ) Théorie des nombres T. 1. art. 119. 
ersten Näherungswerth einer imaginären Wurzel algebraischer oder transcendenter Gleichungen $\mathrm{zu}$ finden. Ist nemlich die Gleichung $\boldsymbol{F} x=0$ gegeben, so setzt Legendre $x=\alpha+\beta \checkmark-1$, wo $\alpha$ und $\beta$ beliebige reelle Zahlen sind, und substituirt diesen Werth in $F(x)$, so dafs $F(\alpha+\beta \checkmark-1)=$ $\boldsymbol{P}+\boldsymbol{Q} \checkmark-1$ ist, wo $\boldsymbol{P}$ und $\boldsymbol{Q}$ wieder reelle Gröfsen sind. Ferner substituire man diese Werthe von $x$ in $\frac{\partial F x}{\partial x}$ und es sei unter dieser Voraussetzung $\frac{\partial F x}{\partial x}$ $=\boldsymbol{M}+\boldsymbol{N} \checkmark-1$. Nimmt man nun eine reelle oder imaginäre unbestimmte Gröfse $\omega$, die aber im Verhältnifs zu $\sqrt{ }\left(\alpha^{2}+\beta^{2}\right)$ sehr klein ist, so hat man, wenn man $x=\alpha+\beta \checkmark-1+\omega$ setzt und die höheren Potenzen von $\omega$ vernachlässigl,

$$
\boldsymbol{F}(\alpha+\beta r-1+\omega)=\boldsymbol{P}+\boldsymbol{Q} \dot{r}-1+\omega(M+N r-1) .
$$

Da nun $\omega$ willkürlich ist, so kann man

$$
\omega(M+N \checkmark-1)=-n(P+Q \checkmark-1)
$$

setzen, wo $n$ ein ächter Bruch ist. Man hat also einen neuen Nälerungswerth

$$
F(x)=(1-n)(P+Q \curlyvee-1),
$$

welcher in Verhältnifs von $1-n$ zu 1 kleiner ist als der frühere Näherungswerth. Fährt man auf diese Weise fort, indem man wieder $x$ um eine unbestimmte reelle oder imaginäre Grö Ise wachsen lärst, deren Werth man nachher genauer bestimmt, so kann man sich dem wahren Werthe von $x$ immer mehr nähern. Würde $\frac{\partial F x}{\partial x}$ durch die Substitution eines Werthes von $x=\alpha+\beta \checkmark-1$ auf Null reducirt, so müfste man, statt dieser Function, die Function $\frac{\partial^{2} F x}{\partial x^{2}}$ betrachten und ïberhaupt murs man sich, wenn mehrere der Functionen $\frac{\partial F x}{\partial x}, \frac{\partial^{2} F x}{\partial x^{2}}, \ldots$ durch einen Werth $x=\alpha+\beta r-1$ anf Null reducirt werden, an den ersten Differentialquotienten halten, der nicht Null wird. Auf diese Weise glaubt Legendre zu beweisen, dafs jede algebraische oder transcendente Gleichung eine Wurzel von der Form $\alpha+\beta \sqrt{ }-1$ hat, wo $\beta$ auch Null sein kann. Dieses Resultat ist aber nicht richtig, und es lärst sich auch leicht zeigen, dars Legendre's Verfahren mit mehreren bedeutenden Irrthimern behaftet ist. Legendre selbst hat schon eine Mangelhaftigkeit desselben bemerkt *), welche darin besteht, dafs man den ersten Näherungswerth gana willkürlich annimmt, so dafs dieser hypothelische Werth vom wahren Werthe

*) Thóar. des nombres ed 3. T. II. p. 420. 
bedeutend abweichen kann und man daher nothweudig sehr weitläuftige Rechnungen machen mufs. Dies ist aber nur eine Unbequemlichkeit. Unrichtig ist es dagegen, dafs Legendre bei der Entwicklung von $F(\alpha+\beta \checkmark-1)$ nur die zwei ersten Glieder berücksichtigt, die vermittelst der Taylor'schen Reihe gefunden werden, und den Rest ganz vernachlässigt. Dieses Verfahren, welches der Newton'schen Approximationsmethode ähnlich ist, leidet auch an demselben Fehler, indem die successiven Näherungswerthe, statt gegen den wahren Werth zu convergiren, auch divergiren können, da man Glieder vernachlässigt, deren Werth man nicht kennt. Ferner kann es aber auch sein, dafs die Substitution von $\alpha+\beta \sqrt{ }-1$ für $x$ einen der Differentialquotienten auf $z$ reducirt, und alsdann hört nalürlich die Brauchbarkeit des Verfalirens ganz auf.

Diese Fehler hat Cauchy dadurch vermieden, dafs er nicht eine beliebige Function $\boldsymbol{F}(\boldsymbol{x})$ betrachtet, sondern die Untersuchung auf Functionen beschränkt, welche nebst ihren sämntlichen Differentialquotienten für alle endlichen reellen oder imaginären Werthe von $x$ endlich und stetig bleiben, und unendlich grofs werden, wenn der Modulus der Veränderlichen $x$ unendlich grofs wird. Cauchy zeigt alsdann, wie man bei solchen Functionen uach Legendre's Verfahren unter gewissen Voraussetzungen Näherungswerthe finden und die Grenzen der begangenen Fehler bestimmen kann. Mithin ist diese Methode keine allgemein gültige. Ich begnüge mich daher, uur Folgendes darüber zu bemerken. Vermittelst dieser Methode will man sowohl die imaginären als die reellen Wurzeln finden. Soll die Wurzel reell sein, so mufs der imaginäre Theil des gefundenen Werthes verschwinden. Hat nun aber die Gleichung reelle Wurzeln und man hat durch fortgeseczte Annäherung einen Werth $\alpha+\beta \sqrt{ }-1$ gefunden, in welchem $\beta$ sehr klein ist, so kann man immer noch uicht wissen, ob $\beta$ eigentlich gleich Null and also eine reelle Wurzel der Gleichung $=\alpha$ ist, oder ob die Gleichung eine imaginäre Wurzel $\alpha+\beta \sqrt{ }-1$ hat, in welcher $\beta$ sehr klein ist. Grade die reellen Wurzeln, welche vom wichtigsten Interesse sind, köunen also nach dieser Methode am wenigsten sicher gefunden werden.

4. In der neuesten Zeit haben wir durch Fourier eine Methode zur Auflösung der algebraischen Gleichungen erhalten, welche, insofern man diese Auflösung auf die Bestimmung des Werthes der reellen Wurzeln und das Erkennen der imaginären einschränkt, vollkommen genügt. Fourier hat nun mehrfach die Behauptung ausgesprochen, dafs seine Methode nicht 
1. Stern; über die Auflösung der transcendenten Gleichungen.

auf die algebraischen Gleichungen beschränkt sei, sondern auch auf die transcendenten angewandt werden könne. Es sollte sogar das leider nicht erschienene fünfte Buch seines Werkes über die Gleichungen, wie wir aus dem Exposé synoptique ersehen, diese Auwendung seiner Methode ausführlich behandeln. Ich werde mich im Folgenden bemühen, mit Hülfe der Andeutungen, die Fourier an melireren Orten, und besonders in dem erwähnten Exposé gegeben hat, diesen Theil seines Werkes wieder herzustellen und zu zeigen, wie man vermittelst der Fourierschen Methode die transcendenten Gleichungen vollständig auflösen kann, und hoffe auf diese Weise der zweiten Anforderung der Societät d. W. Genüge zu leisteu.

\section{Auflösung der transcendenten Gleichungen.}

5. Der Begriff der Wurzel einer algebraischen Gleichung kann auf zweierlei Arten erklärt werden, die ihrem Wesen nach identisch sind. Ist nemlich eine solche Gleichung

$$
\text { 1. } f(x)=0
$$

gegeben, so nennt man jeden Werth von $x$, der statt $x$ in $f x$ substituirt, diese Function auf Null reducirt, eine Wurzel der Gleichung (1.). Aufserdem weifs man aber auch, dals $f(x)$ das Product eimer Anzahl einfacher reeller oder imaginärer Factoren ist, so dafs man

$$
f(x)=\left(x-\alpha_{1}\right)\left(x-\alpha_{2}\right)\left(x-\alpha_{3}\right) \ldots
$$

hat, wo $a_{1}, a_{2}, a_{3} \ldots$ reelle oder imaginäre Gröfsen sind. Da uun $f(x)$ nur dann den Werth Null annehmen kann, wenn einer dieser Factoren verschwindet und unter dieser Voraussetzung gleich Null wird, da das Product der übrigen Factoren immer eine endliche GröIse bleibt, so kann man auch sagen: die Wurzeln der Gleichung $f(x)=0$ sind die Werthe $a_{1}, \alpha_{2}, a_{3}, \ldots$, , welche den einfachen Factoren von $f(x)$ entsprechen. Man kann daher auch behaupten, dals der erste Theil einer algebraischen Gleichung dem Producte der einfachen Factoren gleich ist, die den Wurzeln dieser Gleichung entsprechen. Anders aber ist es bei den transcendenten Gleichungen. Für diese parst nur die erste Definition des Begriffs der Wurzel und man muls sagen: die Wurzel einer transcendenten Gleichung $f(x)=0$ ist jeder Werth von $x$, welcher $f(x)$ auf Null reducirl. Weifs man nemlich, dafs $f(x)=(x-a) F x$ ist, so folgt hierans noch keinesweges, dafs a eine Wurzel der Gleichung $f(x)=0$ ist. Denn cubstituirt man statt $x$ den Werth $a$, so wird zwar $x-a=0$, aber es 
kann sein, dafs zu gleicher Zeit $F(a)=\infty$ wird. Man bătte daher in diesem Falle $f(a)=0 . \infty$, welcher Ausdruck keinesweges immer $=0$ sein mufs. Mithin ist zwar hier $x-a$ ein einfache Factor von $f(x)$, aber dennoch ist a keine Wurzel der Gleichung $f(x)=0$, und es folgt hieraus, dafs bei den transcendenten Gleichungen keinesweges jeder einfache Factor des ersten Theils der Gleichung einer Wurzel entspricht, obwohl umgekehrt jede Wurzel einem einfachen Factor. Ist z. B. die Gleichung

$$
\operatorname{tang} x=0
$$

gegeben, so kann man $\operatorname{tang} x$ als das Product der zwei Faetoren $\sin x$ und sec. $x$ ansehen. Der Factor $\sin x$ ist nun bekanntlich das Product einer unendlicheu Anzahl einfacher Factoren, da

$$
\sin x=x\left(1-\frac{x^{2}}{\pi^{2}}\right)\left(1-\frac{x^{2}}{4 \pi^{2}}\right)\left(1-\frac{x^{2}}{9 \pi^{2}}\right) \ldots
$$

ist; und dieses Product wird Null, sobald man einen der Factoren gleich Null selzt; das heirst, die Wurzeln der Gleichung

$$
\sin x=0
$$

sind $x=0, x=\pi, x=2 \pi, x=3 \pi, \ldots$. Diese Wurzeln sind zugleich Wurzeln der Gleichung $\operatorname{tang} x=0$; denn sobald $\sin x=0$ ist, ist sec. $x=1$, also in diesem Falle tang $x=0.1=0$. Dagegen sind die Wurzeln der Gleichung sec. $x=0$ keinesweges Wurzeln der Gleichung tang $x=0$. Soll nemlich sec. $x$ oder $\frac{1}{\cos x}$ Null werden, so mufs $\cos x$ unendlich grofs sein. So lange aber $x$ eine reelle Gröfse ist, kann dies nicht sein, das heifst, die Gleichung sec. $x=0$ hat keine reelle Wurzel. Setzt man dagegen $x=y+z \sqrt{ }-1$, wo $y$ und $z$ reelle Grölsen sind, so ist $\cos x=$ $\frac{1}{2}\left(e^{z}+e^{-z}\right) \cos y-\frac{1}{2}\left(e^{z}-e^{-z}\right) \sin y \checkmark-1$. Soll nun dieser Ausdruck unendlich grofs werden, so mufs $z$ unendlich grofs sein. In diesem Falle hat man also

$$
\cos x=\frac{1}{2} e^{\infty}(\cos y-\sin y \checkmark-1) .
$$

Nun ist aber, wenn man $x=y+z \sqrt{ }-1$ setzt,

$$
\sin x=\frac{1}{2}\left(e^{z}+e^{-z}\right) \sin y+\frac{1}{2}\left(e^{z}-e^{-z}\right) \cos y \checkmark-1
$$

und, wenn $\operatorname{man} z=\infty$ setzt,

$$
\sin x=\frac{1}{2} e^{\infty}(\sin y+\cos y \gamma-1)
$$

also ist $\sin x=\infty$, wenn $\frac{1}{\cos x}=0$ ist, das heifst : wean man statt = eine Crolle's Journal d. M. Bd. XXII. Hft. 1. 
Wurzel der Gleichung $\sec . x=0$ substituirt, so wird tang $x=0 . \infty$; was nicht nothwendig Null ist. Auch sieht man leicht, dafs unter diesen Umständen $\operatorname{tang} x$ wirklich einen anderen Werth als Null hat. Denn es ist in diesem Falle

$$
\operatorname{tang} x=\frac{\sin x}{\cos x}=\frac{\sin y+\cos y r-1}{\cos y-\sin y r-1}=r-1 * \text {. }
$$

Das Product aller einfachen Factoren, welche den Wurzeln der Gleichung $\operatorname{tang} x=0$ entsprechen, giebt also keinesweges tang $x$, sondern $\sin x$.

6. Es kann auch sein, dafs es transcendente Functionen giebt, welche durch keinen reellen oder imaginären Werth von $x$ auf Null reducirt werden künnen; wiewohl vielleicht noch keine solche Function bis jetzt bekannt ist. In diesem Falle würde also die Gleichung $f(x)=0$ gar keine Wurzel haben. Es giebt zwar viele, sowohl algebraische als transcendéte Functionen, von welchen sich nachweisen lärst, dafs sie durch keinen endlichen reellen oder imaginären Werth von $x$ auf Null reducirt werden können (dahin gehören die Functionen $\frac{1}{x}, e^{x}, e^{x y-1}$ ): dennoch aber darf nicht behauptet werden, dafs z. B. die Gleichung $e^{x}=0$ keine Wurzel habe, indem sich vielmehr nachweisen läfst, dafs sie deren unendlich viele hat, die sämmtlich in der Form $x=-\infty$ enthalten sind. Es wäre aber offenbar eine Einseitigkeit, wenn man die unendlich grofsen Werthe nicht als Wurzeln gelten lassen wollte; auch wird sich später zeigen (\$.31.), dafs die Betrachtung dieser Wurzeln in bestimmten Fällen durchaus nothwendig ist. Eben so hat die Gleichung $e^{x \vee-1}=0$ eine unendliche Zahl von Wurzeln, die sämmtlich in der Form $x=-\infty \sqrt{ }-1$ enthalten sind. Wenn ich aber sage, dafs eine transcendente Gleichung $f(x)=0$ gar keine Wurzeln hat, so verstehe ich darunter, dafs sie weder durch endliche noch durch unendliche Werthe auf Null reducirt werden kann, und es ist bis jetzt nicht ausgemacht, ob solche Functionen vorhanden sein können oder niclit **).

*) Dafs tang $x=V-1$ wird, wenn sec. $x=0$ ist, hat bereits Fourier in einer Abhandlung bewiesen, die jedoch bis jetzt nicht erschienen ist. Man vergleiche Mém. de l'acad. d. sc. T. $X$. pag. 129 ;

*) Auch Fourier drückt sich darübér zweifelhaft aus, indem er sagt (Exposé synopt. $p, 65,9)$ : is Mais b'il pouvait exister un facteur $F x$ qui ne cesserait point d'aroir une valeur finie, quelque valeur réelle ou imaginaire que l'on attribuât à $x$." 
7. Ist mithin eine transcendente Function gegeben (sie heifse $f(x)$ ) und man kennt alle reellen oder imaginären Wurzeln der Gleichung $f x=0$, nemlich $\alpha, \beta, \gamma, \delta \ldots$ und bezeichnet durch $\phi(x)$ das Product

$$
\left(1-\frac{x}{\alpha}\right)\left(1-\frac{x}{\beta}\right)\left(1-\frac{x}{\gamma}\right) \ldots
$$

aller einfachen Factoren, welche den Wurzeln der Gleichung $\varphi(x)=0$ entsprechen, so kann es sein, dals dieses Product nicht $=f(x)$ ist. Es kanu nemlich, wenn $f x=\phi_{x} \cdot \boldsymbol{F} x$ ist, der Factor $F x$ so beschaffen sein, dafs er nur durch solche Werthe von $x$ auf Null reducirt wird, die, in $\boldsymbol{\varphi}_{\boldsymbol{x}}$ substituirt, diese Function auf $\propto$ reduciren, so dafs die Wurzeln von $\boldsymbol{F} \boldsymbol{x}$ nicht zugleich Wurzeln von $f x$ sind; oder es kann auch $F x$ eine Function sein, die durch keinen reellen oder imaginären Werth von $x$ auf Null gebracht wird $\left.{ }^{*}\right)$. Lä(st sich dagegen die Function $f x$ in einfache Factoren zerlegen, die den Wurzeln der Gleichung $f x=0$ entsprechen, so dals mau

$$
f x=\left(1-\frac{x}{\alpha}\right)\left(1-\frac{x}{\beta}\right)\left(1-\frac{x}{\gamma}\right) \ldots
$$

hat und diese Factoren so beschaffen sind, dafs, wenn man einen derselben gleich Null setzt und den daraus entspringenden Werth von $x$ in die übrigen Factoren substituirt, keiner dieser übrigen Factoren unendlich grofs wird, so kann die Gleichung keine anderen Wurzeln als $\alpha, \beta, \gamma$.... haben. Dewn substituirt man statt $x$ irgend einen anderen Werth, so kann dieser keinen der Factoren, aus welchen $f x$ besteht, und also auch $f x$ selbst nicht auf Null reduciren.

8. Das Aufsuchen der reellen Wurzeln einer transcendenten, wie einer algebraischen Gleichung, geschieht dadurch, dafs man Grenzen sucht, zwischen welchen die einzelnen Wurzeln enthalten sind und dals man diese Grenzen inmer enger zusammenzieht. Eine algebraische Function $f x$ ist aber immer eine continuirliche, das heifst, eine Function, die nur um ein unendlich Kleines wächst, wenn die Veränderliche $x$ einen unendlich kleinen Zuwachs erhält. Der Werth einer solchen Function kann daher nicht vom Positiven zum Negativen und ungekehrt übergehen, ohne dazwischen Null zu werden. Eine transcendente Function $f x$ dagegen kann auch eine discontinuirliche sein, das heifst, es können Grenzwerthe von $x$ vorkommen, die so beschaffen sind, dafs einem unendlich kleinen Zuwachse von $x$ ein

*) Es versteht sich von selbst, dafs das Product der den Wurzeln entsprechenden einfachen Factoren einer Gleichung $A \cdot f x=0$, wenn $A$ eine Constante ist, nicht $A . f s$, sondern nur $f \boldsymbol{x}$ werden kann. 
endlicher oder unendlich grofser Zuwachs von $f \boldsymbol{x}$ entspricht. $\mathbf{Z}$ wischen solchen zwei benachbarten Grenzwerthen, bei welchen die Continuität aufhört, wird also die transcendente Function continuirlich sein. Sucht man daher die Wurzeln einer transcendenten Gleichung $f x=0$, so muls man zuerst sehen, ob $f x$ eine continuirliche oder eine discontinuirliche Function ist. Diese Untersuchung gehört nicht in das Gebiet der Theorie der Gleichungen. Dieselbe selzt sie viehmehr voraus. Hat man gefunden, dafs $f x$ eine discontinuirliche Function ist, und kennt man die Grenzwerthe, bei welchen die Continuität aufhört, so betrachtet man, stalt der Funclion in Allgemeinew, die einzelnen Theile derselben, welche zwischen je zwei Grenzwerthen enthalten sind und sucht die in diesen $\mathbf{Z}$ wischenräumen enthaltenen Wurzeln. Insofern man daher voraussetzt, wie es im Folgenden immer geschieht, dafs man die in den transcendenten Gleichungen vorkommenden Functionen nur innerhalb der Grenzen betrachtet, zwischen welchen sie continuirliche Grölsen sind, gilt auch für diese Gleichungen folgender Lehrsatz.

"Wenn die Gleichung $f x=0$ gegeben ist, und man substituirt statt $x$ die Werthe $x_{1}$ und $x_{2}$, so werden, wenn $f x_{1}$ und $f x_{2}$ entgegengesetzte Zeichen haben, eine oder mehrere Wurzeln dieser Gleichung zwischen den Grenzen $x_{1}$ und $x_{2}$ enthalten sein. Liegt keine reelle Wurzel der Gleichung zwischen diesen Grenzen, so haben $f x_{1}$ und $f x_{2}$ gleiche Zeichen."

Der Beweis dieses Satzes ist bekannt und folgt unmittelbar aus dem Begriffe der Continuität.

9. Ein anderer, ebenfalls bekannter Satz, von welchem später häufig Gebrauch gemu: ht werden wird, ist folgender.

„Weun eine Function $f x$, so wie auch ihre Differentialquotienten $\frac{\partial f x}{\partial x}, \frac{\partial^{2} f x}{\partial x^{2}}, \frac{\partial^{3} f x}{\partial x^{3}} \ldots$ zwischen den Grenzen $x$ und $x+\alpha$ continuirliche Gröfsen sind, so hat man

$$
\begin{aligned}
& f(x+a)=f x+\frac{a \cdot \partial f(x, x+a)}{\partial x}, \\
& f(x+a)=f x+\frac{a \cdot \partial f x}{\partial x}+\frac{a^{2}}{2} \frac{\partial^{2} f(x, x+a)}{\partial x^{2}} \\
& f(x+a)=f x+\frac{a \cdot \partial f x}{\partial x}+\frac{a^{2}}{2} \frac{\partial^{2} f x}{\partial x^{2}}+\frac{a^{3}}{2.3} \frac{\partial^{3} f(x, x+a)}{\partial x^{3}} \\
& \quad \text { etc. etc. }
\end{aligned}
$$

wo $(x, x+a)$ eine Gröfse bedeutet, die zwischen $x$ und $x+a$ enthalten ist, aber in den verschiedenen Gleichungen verschiedene Werthe haben 
kann. Den Beweis dieses Satzes findet man z. B. in Lagrange Lecons sur le calc. des fonct. lec. 9. Err gilt auch für den Fall, wenn $f x$ eine continuirliche imaginäre Function ist.

10. Nach diesen Vorbereitungen will ich nun zuerst zeigen, wie man die Grenzen finden kann, zwischen welchen die reellen Wurzeln der transcendenten Gleichungen enthalten sind. Wiewolıl ich die Untersuchungen Fourier's über die Theorie der algebraischen Gleichungen als bekannt voraussetzen muls, will ich doch zuerst, zur deutlicheren Einsicht, in der Kürze das Verfahren andeuten, vermittelst dessen er die Grenzen der reellen Wurzeln findet.

Ist eine algebraische Gleichung

$$
f x=x^{m}+a_{1} x^{m-1}+a_{2} x^{m-2}+\ldots+a_{m}=0
$$

gegeben, so bilde man die successiven Differenzialquotienten $f^{1} x, f^{2} x$, $f^{3} x, \ldots$ Der letzte Differentialquotient $f^{m} x$ ist eine constante Gröfse. Nimmt man statt $x$ irgend eine Zahl $a$ und substituirt dieselbe in die Ausdrücke

$$
f^{m} x, f^{m-1} x, \ldots f^{1} x, f x,
$$

so werden die sich hieraus ergebenden Werthe das Zeichen von Positiv oder Negativ vor sich haben. Diese Zeichen schreibe man in der Ordnung, wie man sie erhält, neben einander, in eine horizontale Reihe, von der Linken zur Rechten fortgehend, und bezeichne die Reihe von Zeichen durch [a].

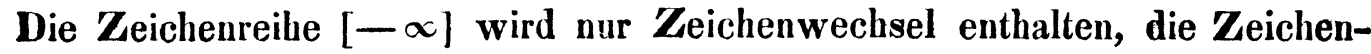
reihe $[\infty]$ nur Zeichenfolgen; und zwar verliert die Zeichenreihe ihre Zeichenwechsel beim Uebergange von $-\infty \mathrm{zu} \infty$ allmälig, ohne jemals einen Zeichenwechsel, den sie verloren hat, wieder zu erhalten, oder neue zu bekommen. Sobald man näılich statt $x$ eine reelle Zahl $\alpha$ substituirt, welche $f x$ auf Null reducirt, so verliert die Zeichenreihe einen Zeichenwechsel, den sie nicht wieder erhält. Sie kann aber auch Zeichenwechsel dadurch verlieren, dafs einer oder mehrere der Differentialquotienten Null wird, ohne dafs $f x$ Null wird. In diesem Falle verliert sie aber die Zeichenwechsel immer paarweise, und der Verlust eines jeden solchen Paares von Zeichenwechseln deutet auf zwei imaginäre Wurzeln. Man besitzt nun Mittel, um zu unterscheiden, ob der Verlust der Zeichenwechsel von reellen oder imaginären Wurzeln berührt. Sind daher $\alpha$ und $\beta$ zwei reelle Zahlen, ist $\beta>\alpha$ und man findet, dafs $[\alpha] n$ Zeichenwechsel mehr enthält als $[\beta]$, so werden zwischen den Grenzen $\alpha$ und $\beta$ $n$ Wurzeln angedeutet und man kann alsdann untersuchen, ob und wie 
viele reelle Wurzeln wirklich zwischen diesen Grenzen liegen, oder ob der Verlust der Zeichenwechsel ganz oder theilweise von imaginären $W$ urzeln herrührt.

11. Die besondere Eigenthümlichkeit einer ganzen algebraischen Function $f x$ besteht darin, dafs man durch fortgesetzte Differentiation zuletzt zu einem Differentialquotienten kommt, der einen coustanten Werth hat; und vermöge dieser Eigenthümlichkeit ist es möglich, jedesmal die Anzahl der Zeichenwechsel in den zwei Zeichenreihen [a] und $[\beta]$ zu bestimmen und hieraus zu schliefsen, wie viel Wurzeln zwischen den Grenzen $\alpha$ und $\beta$ enthalten sind. Bei den transcendenten Functionen dagegen kann man die Differentiation in's Unendliche fortsetzen, und kommt nie zu einem constanten Werthe. Eben deswegen kann man bei den transcendenten Gleichungen nicht ohne Weiteres bestimmen, wie viele reelle Wurzeln zwischen zwei beliebig geüühlten Grenzen enthalten siud, wenn auch die transcendente Function, welche den ersten Theil der Gleichung bildet, zwischen diesen Grenzen continuirlich ist. Dennoch aber kann man auch bei diesen Gleichungen, auf ganz ähnliche Weise wie bei den algebraischen, alle reelle Wurzeln, die zwischen $-\infty$ und $\infty$ enthalten sind, mit Bestimmtheit entdecken, sobald man nur diesen $\mathbf{Z}$ wischenraum in kleinere $\mathbf{Z}$ wischenrăume theilt, die nach einem bestimmten Gesetze gebildet werden müssen. Man kann nemlich für jede transcendente Function $f x$ einen Differentialquotienten $f^{m} x$ findeu, der so beschaffen ist, dafs er zwischen zwei Grenzen $\alpha$ und $\beta$ sein Zeichen nicht ändert, dafs also die Gleichung $f^{m} x=0$ zwischen diesen Grenzen keine Wurzel hat; und dieser Differentialquotient spielt in Beziehung auf diese Grenzen dieselbe Rolle, wie der constante Differentialquotient bei den algebraischen Gleichungen, indem man vermittelst desselben bestimmen kann, wie viele Wurzeln die transcendente Gleichung $f x=0$ zwischen den Grenzen $\alpha$ und $\beta$ hat. Dafs man wirklich jedesmal einen solchen Differentialquotienten finden kann, ist klar. Denn die gegebene Gleichung $f x=0$ ist nothwendig eine bestimmte, das leifst, die Function $f x$ ist von der Art, dafs man aus ihr für jeden Werth $\gamma$, den man statt $x$ substituirt, den Werth von $f(\gamma)$ mit Bestimmtheit finden kann, sei es nun, dafs man ihn genau angeben, oder in beliebig enge Grenzen einschliefsen kann; was z. B. der Fall ist, weun $f x$ eine convergirende Reile ist. Wäre $f x$ eine unbestimmte Function, so könnte natürlich von der Auflösang der Gleichung $f x=0$ nicht die Rede sein. 
Es müssen mithin auch die successiven Differentialquotienten von $f x$ nothwendig bestimmte Functionen sein. Man wird also aus der Natur irgend eines Differentialquotienten erkennen können, ob er für einen bestimmten Werth $x=\alpha$, positiv oder negativ ist, und da dieser Differenzialquotient, wie hier immer vorausgesetzt wird, zwischen bestimmten Grenzen eine continuirliche Grörse ist, so wird man immer einen Zuwachs $\delta$ von $x$ bestimmen können, so beschaffen, dafs der Differentialquotient, welcher $f^{m} x$ heifsen mag, innerhalb der Grenzen $x=\alpha, x=\alpha+\delta$, immer dasselbe Zeichen behält. Sobald diese Grenzen gefüunden sind, kann man, auf dieselbe Weise wie bei den algebraische!. Gleichungen, bestimmen, wie viele Wurzeln die gegebene Gleishlung zwischen diesen Grenzen hat. Es mufs nur eine Modification in Beziehung auf die Bildung der Zeichenreihen eintreteu. Bei den äigebraischen Gleichungen bildet man die Zeichenreihe, indem man zuerst alle Differentialquotienten, vom letzten anfangend, in eine Reihe schreibt, und alsdann statt $x$ den Werth $\alpha$ substituirt. Die hieraus entspringende Zeichenreihe wurde oben durch $[\alpha]$ bezeichnet. Bei den transceudenten Gleichungen sucht man zuerst einen Differentialquotienten, der zwischen zwei Grenzen $\alpha$ und $\beta$ dasselbe Zeichen behält ${ }^{*}$ ). Schreibt man diesen Differentialquotienten, und alle folgenden, nach der Ordnung in eine horizontale Linie und substituirt alsdann statt $x$ einen Werth $a$, der zwischen $\alpha$ und $\beta$ liegt, in alle diese Functionen, so erhält man wieder eine Reihe von Zeichen, die im Allgemeinen theils positiv, theils negativ sein werden. Diese Zeichenreihe soll im Folgenden durch $[a]$ bezeichnet werden. Ich werde auch zur Abkürzung die zwei Grenzen, zwischen welchen ein bestimmter Differentialquotient sein Zeichen nicht ändert, die bestimmenden Grenzen und diesen Differentialquotienten den bestimmenden nennen.

12. Liegt eine Zahl $a$ zwischen den bestimmenden Grenzen $\alpha$ und $\beta$, so ist es einleuchtend, dafs die Zeichenreihe [a] mit der Zeichenreihe [ $\alpha]$ identisch ist, so lange nicht zwischen $\alpha$ und $a$ eine Zahl liegt, die, statt $x$ substituirt, eine oder mehrere der auf den bestimmenden Differentialquotienten folgenden Functionen auf Null reducirt. Denn eine Aenderung in der Zeichenreihe kaun nur dadurch entstehen, dafs eine oder mehrere die-

*) Es wird im Folgenden immer vorausgesetzt, dafs $\beta$, mit Rücksicht auf das Zeichen, immer grölser ist als $\alpha$. 
ser Functionen vom Positiven zum Negativen, und umgekehrt, übergehen; und da vorausgesetzt wird, dafs alle Functionen zwischen den bestimmenden Grenzen continuirlich sind, so kann dieser Uebergang nicht Statt haben, wenn die Functionen nicht dureh den Werth Null gehen. Man nehme daher zuerst an, die Zahl $a$ sei so beschaffen, dafs sie nur $f x$ und keine der übrigen Functionen auf Null reducirt, so dafs also $a$ eine Wurzel der Gleichung $f x=0$ ist. Man setze in allen Functionen statt $x$ nach einander die drei Werthe $a-\partial a, a, a+\partial a$ und schreibe die hierdurch entstehenden drei Zeichenreiben $[a-\partial a],[a],[a+\partial a]$ auf drei horizontale Linien unter einander. Diese drei Zeichenreihen werden nur in Absicht auf das letzte Zeichen verschieden sein; denn da der Werth von da ganz unbestimnt ist und nach der Yoraussetzung die Substitution von $a$ für $x$ nur $f x$ auf Null reducirt, so kann man ihn immer so klein annehmen, dafs keine der übrigen Functionen ihr Zeichen zwischen den Grenzen $a-\partial a$ und $a+\partial a$ ändert. Es wird aber $f(a+\partial a)$ positiv oder negativ und $f(a-\partial a)$ negativ oder positiv sein, je nachdem $f^{\prime} a$ positiv oder negativ ist. Ist $f^{\prime} a$ positiv, so lat man das Schema

$$
\begin{aligned}
{[a-\partial a] } & =\ldots+- \\
{[a] } & =\ldots+0 \\
{[a+\partial a] } & =\ldots++
\end{aligned}
$$

Ist $f^{\prime} a$ negativ, so hat man das Schema

$$
\begin{aligned}
{[a-\partial a] } & =\ldots .+ \\
{[a] } & =\ldots-0 \\
{[a+\partial a] } & =\ldots .-
\end{aligned}
$$

In beiden Fällen verliert also die Zeichenreihe einen Zeichenwechsel beim Uebergange von $a-\partial a$ zu $a+\partial a$, sobald $a$ eine Wurzel der Gleichung $f x=0$ ist.

13. Ist dagegen die zwischen den bestimmenden Grenzen enthaltene Zahl a so beschaffen, dafs sie eine der auf den bestimmenden Differentialquotienten folgenden Functionen, z. B. $f^{n} x$, und nur diese auf Null reducirt, so werden auch in diesem Falle alle Zeichen der drei Reihen $[a-\partial a]$, [a] und $[a+\partial a]$ bezüglich gleich sein, bis auf dasjenige, welches durch die Substitution der drei Werthe $a-\partial a, a$ und $a+\partial a$ in $f^{n} x$ entsteht, und man hat daher nur nöthig, die drei auf einander folgenden Functionen $f^{n+1} x$, $f^{n} x, f^{n-1} x$ zu betrachton, wenn man wissen will, wie sich die drei Reihen $[a-\partial a],[a]$ und $[a+\partial a]$ gegen einander verhalten. Die Fumction 
$f^{n}(a-\partial a)$ ist negaliv oder positiv, die Fanction $f^{n}(a+\partial a)$ positiv oder negativ, je nachdem $f^{n+1} a$ positiv oder negativ ist. Die Function $f^{n-1} a$ kann aber ebenfalls positiv oder negativ sein. Hierdurch entstehen vier verschiedene Combinationen. Sind nemlich $f^{n+1} a$ und $f^{n-1} a$ positiv, so hat man, wenn man nur die Zeichen der Functionen $f^{n+1} x, f^{n} x, f^{n-1} x$, schreibt :

$$
\begin{aligned}
{[a-\partial a] } & =\ldots .+\ldots++\ldots \\
{[a] } & =\ldots .+0+\ldots \\
{[a+\partial a] } & =\ldots .+\ldots++\ldots
\end{aligned}
$$

Sind $f^{n+1} a$ und $f^{n-1} a$ beide negativ, so hat man

$$
\begin{array}{r}
{[a-\partial a]=\ldots .+\ldots} \\
{[a]=\ldots .0-\ldots .} \\
{[a+\partial a]=\ldots .-\ldots}
\end{array}
$$

Ist $f^{n+1} a$ negativ und $f^{n-1} a$ positiv, so hat man

$$
\begin{aligned}
{[a-\partial a] } & =\ldots--+\ldots \\
{[a] } & =\ldots .0+\cdots \\
{[a+\partial a] } & =\ldots .+\ldots
\end{aligned}
$$

Ist $f^{n+1} a$ positiv und $f^{n-1} a$ negativ, so hat man

$$
\begin{aligned}
{[a-\partial a] } & =\ldots+ \\
{[a] } & =\ldots+\ldots+0-\ldots \\
{[a+\partial a] } & =\ldots+\ldots+-\ldots
\end{aligned}
$$

In den zwei Fällen, wenn $f^{n+1} a$ und $f^{n-1} a$ gleiche Zeichen haben, enthält also $[a+\partial a]$ zwei Zeichenwechsel weniger als $[a-\partial a]$. In den zwei Fällen dagegen, wenn diese Functionen verschiedene Zeichen haben, entbält $[a+\partial a]$ eben so viele Zeichenwechsel als $[a-\partial a]$.

14. Es ist noch der Fall übrig, wenn die Zahl $a$ so beschaffen ist, dafs sie, statt $x$ substituirt, mehrere auf einander folgende der zu betrack tenden Functionen auf Null reducirt. Da indessen die Untersuchung dieses Falles ebenfalls ganz auf dieselbe Weise ausgeführt werden kann wie bei den algebraischen Gleichungen, für welche sie schon Fourier aunführlich angestellt hat, so will ich nur das Resultat hersetzen, wie es in Beziehung auf die transcendenten Gleichungen ausgesprochen werden mufs. Man nehme an, es sei eine zwischen den bestimmenden Grenzen $\alpha$ und $\beta$ liegende Zahl so beschaffen, dafs sie, statt $x$ substituirt, die $i$ auf einander folgenden Functionen

$$
f^{n} x, f^{n-1} x, \ldots f^{n-i+1} x
$$

Crelle's Journal d. M. Bd. XXII. Hif. 1. 
auf Null reducirt, so sind $z w e i$ Fälle zu unterscheiden. Ist $i$ eine gerade Zahl, so enthält $[a+\partial a]$ die $Z$ ahl $i$ von Zeichenwechseln weniger als $[a-\partial a]$; ist aber $i$ eine ungerade $\mathbf{Z a h l}$, so kommt es darauf an, ob $f^{n+1} a$ und $f^{n-i}$ gleiche oder ungleiche Zeichen haben. Im ersten Falle hat $[a+\partial a] i+1$, im zweiten $i-1$ Zeichenwechsel weniger als $[a-\partial a]$ Verschwindeu nun durch die Substitution eines Werthes $a$ von $x$, an verschiedenen Stellen verschiedene Gruppen von Functionen, so dafs die Anzahl der verscliwindenden Functionen an einer Stelle $i$, an einer anderen $i^{\prime}$ u. s. w. beträgt, so braucht man nur für jede Gruppe die Regeln in Anwendung zu bringen, die so eben für eine einzelue Gruppe gegeben worden sind und findet auf diese Weise die Totalsumme der Zeichenwechsel, die $[a+\partial a]$ weniger enthält als $[a-\partial a]$.

15. Aus dem Vorhergehenden ergiebt sich deutlich, wie man die Zahl finden kann, welche angiebt, wie viele reelle Wurzeln der Gleichung $f x=0$ zwischen den bestimmenden Greuzen $\alpha$ und $\beta$ liegen können. So lange nemlich zwischen $\alpha$ und $\beta$ keine Zahl liegt, welche eine oder mehrere der zu betrachtenden Functionen auf Null reducirt, sind die Zeichenreihen [ $\alpha]$ und $[\beta]$ identisch. Diese Zeichenreihen könuen nur dann von einander verschieden sein, wenn die Function $f x$, oder einer ihrer Differentialquotienten zwischen den Grenzen $\alpha$ und $\beta$ Null wird. In diesem Falle mufs aber die Zeichenreihe $[\beta]$ immer weniger Zeichenwechsel als die Reihe [a] haben, indem, wenn man den Werth von $x$ wachsen läfst, nur Zeichenwechsel verschwinden, nie aber die verlorenen wieder erscheinen, oder neue hiuzu kommen können.

Liegen $n$ reelle Wurzeln zwischen $\alpha$ und $\beta$, so mufs [ $\beta$ ] wenigstens $n$ Zeichenwechsel weniger als $[\alpha]$ entbalten. Denn hezeichnet man die Wurzeln, nach ihrer Gröfse geordnet, durch $\alpha_{1}, \alpha_{2}, \alpha_{3} \ldots$. , so hat $\left[\alpha_{1}+\partial \alpha_{1}\right]$ wenigstens einen Zeichenwechsel weniger als $[\alpha]$; eben so $\left[\alpha_{2}+\partial \alpha_{2}\right]$ wenigstens einen Zeichenwechsel weniger als $\left[a_{1}+\partial a_{1}\right]$ u.s. w.

Umgekehrt darf man aber aus dem Umstande, dafs $[\beta]$ die 'Lahl $n$ von Zeichenwechseln weniger enthält als $[\alpha]$, nicht schliefsen, dafs zwischen $\alpha$ und $\beta$ wirklich $n$ reelle Wurzeln liegen, weil das Verschwinden der Zeichen auch daraus entstehen kann, dafs einer oder mehrere der Differentialquotienten von $f x$ durch die Substitution eines zwischen $\alpha$ und $\beta$ liegenden Werthes auf Null reducirt werden, ohne dafs dies bei $f x$ der Fall wäre. Soviel aber ist gewifs, dafs, wenn $n$ eine ungerade Zahl ist, zwischen $a$ 
und $\beta$ wenigstens eine reelle Wurzel liegt, weil, wenn einer oder mehrere der Differentialquotienten Null werden, entweder gar kein Zeichenwechsel, oder eine gerade Zahl von Zeichenwechseln verischwindet.

16. Der Zweifel, ob der Verlust der Zeichenwechsel auf reelle Wurzeln deute, die zwischen den bestimmenden Grenzen $\alpha$ und $\beta$ enthalten sind, oder ob er davon herrühre, dafs einer oder mehrere der. Differentialquotienten zwischen diesen Grenzen Null, werden, kann bei den transcendenten Gleichungen durch dieselbe Regel gelöset werden, die Fourier für den ähulichen Fall bei den algebraischen Gleichungen gegeben hat. Ich werde hier aber um so lieber einen analytischen Beweis hersetzen, der auf die transcendenten und algebraischen Gleichungen zugleich anwendbar ist, als Fourier diese Regel durch geometrische Betrachtungen erwiesen hat.

Man betrachte zuerst den Fall, wenn $[\alpha]$ nur zwei Zeichenwechsel mehr als $[\beta]$ hat, und setze zugleich voraus, dafs dieser Unterschied sich erst in den zwei letzten Zeicben jeder Reihe zeigt, so dafs die vorhergehenden, sich entsprechenden Zeichen in beiden Reihen dieselben sind, so ist ein doppeltes Schema möglich. Entweder ist

oder

$$
\begin{aligned}
& {[\alpha]=\ldots++-+} \\
& {[\beta]=\ldots+++}
\end{aligned}
$$

$$
\begin{aligned}
& {[\alpha]=\ldots \ldots-+-} \\
& {[\beta]=\ldots \ldots--}
\end{aligned}
$$

In beiden Fällen hat $f^{2} x=0$ keine Wurzel, die zwischen $\alpha$ und $\beta$ enthalten ist, weil, sobald man die zwei letzten Zeichen vernachlässigt, die Zeichenreihe $[\alpha]$ nicht mebr Zeichenwechsel hat als die Zeichenreihe [ $\beta$ ]. Man kann daher $f^{2} x$ als die bestimmende Function annehmen. Dagegen hat $f^{1} x=0$ eine Wurzel zwischen diesen Grenzen und es entsteht nun die Frage ob $f x=0$ zwei reelle Wurzeln oder keine zwischen diesen Grenzen habe. Es wird bierbei vorausgesetzt, dafs man sich schon versichert hat, die Gleichung $f x=0$ habe nicht zwei gleiche Wurzeln zwischen $\alpha$ und $\beta$, das heifst, dafs man untersucht hat, ob $f x$ und $f^{1} x$ einen gemeinschaftlichen Factor haben und ob dieser gemeinschaftliche Factor, weun er existirt, eine Wurzel zwischen $\alpha$ und $\beta$ habe.

Man betrachte zunächst das erste Schema. Man sieht sogleich, dars, weun $a$ die Wurzel der Gleichung $f^{1} x=0$ ist, die zwischen $\alpha$ und $\beta$ liegt, alsdaun die Zeichenreihe $[a]$ mit $+0-$ schliefsen mufs, sobald zwischen 
$\alpha$ und $\beta$ zwei reelle Wurzeln der Gleiohung $f x=0$ liegen sollen, weil in dem anderen Falle, der hier noch möglich ist, weun nemlich [a] mit +0 + schliefst, hieraus ron selbst das Nichtvorbandensein der reellen Wurzeln folgt. Denn im letzteren Falle hätte mạn.

$$
\begin{aligned}
& {[a-\partial a]=\ldots+++} \\
& {[a+\partial a]=\ldots+++}
\end{aligned}
$$

Die zwei Zeichenwechsel würden also zwischen den Grenzen $a-\partial a$ und $a+\partial a$, zwischen welchen nach der Voraussetzung keine reelle Wurzel der Gleichung $f x=0$ liegt, verloren gehen. Mithin mufs man, wenn die zwei reellen Wurzeln vorhanden sind, nothwendig folgendes Schema haben:

$$
\begin{aligned}
{[a] } & =\ldots++-+ \\
{[a-\partial a] } & =\ldots++- \\
{[a+\partial a] } & =\ldots++ \\
{[\beta] } & =\ldots+++
\end{aligned}
$$

and es ist eine reelle Wurzel zwischen $\alpha$ und $a-\partial a$, die andere zwischen $a+\partial a$ und $\beta$ enthalten. Es sei die kleinere Wurzel $x_{1}=\alpha+b$, die gröfsere $x_{q}=\beta-b^{\prime}$. Hieraus folgt (9)

$$
f(\alpha+b)=f \alpha+b f^{\prime}(\alpha, \alpha+b)=f \alpha+\left(x_{1}-\alpha\right) f^{\prime}(\alpha, \alpha+b)=0
$$

oder

Auf dieselbe Weise findet man

$$
x_{1}=\alpha-\frac{f a}{f^{\prime}(\alpha, \alpha+b)} \text {. }
$$

$$
x_{2}=\beta-\frac{f \beta}{f^{\prime}\left(\beta-b^{\prime}, \beta\right)} .
$$

Da $f^{\prime} x$ zwischen den Grenzen $\alpha$ und $\beta$ continuirlich ist, so bleibt der Werth dieser Function negativ, so lange man für $x$ eine Zahl substituirt, die zwischen a und a liegt; und zwar wird der numerische Werth der Function kleiner, je näher die statt $x$ substiluirte Zahl dem Werthe $a$ kommt. Aus demselben Grunde bleibt $f^{\prime} x$ positiv, so lange man statt $x$ eine Zahl substituirt, die zwischen $a$ und $\beta$ liegt, und der Werth dieser Function wächst, je mehr sich die statt $x$ substituirte $Z$ ahl dem Werthe $\beta$ nähert. Es ist also (ohne Rücksicht auf das Zeichen)

folglich

$$
\begin{aligned}
& f^{\prime}(\alpha, \alpha+b)<f^{\prime} \alpha \\
& f^{\prime}\left(\beta-b^{\prime}, \beta\right)<f^{\prime} \beta,
\end{aligned}
$$

$$
\begin{aligned}
& x_{1}>\alpha-\frac{f \alpha}{f^{\prime} \alpha} \\
& x_{2}<\beta-\frac{f \beta}{f^{\prime} \beta},
\end{aligned}
$$


und um so mehr

$$
\alpha-\frac{f \alpha}{f^{\prime} \alpha}<\beta-\frac{f \beta}{f^{\prime} \beta}
$$

da $x_{1}$ kleiner als $x_{2}$ ist, das heirst, es mufs

sein.

$$
\frac{f \beta}{f^{\prime} \beta}+\frac{f \alpha}{-f^{\prime} \alpha}<\beta-\alpha
$$

Dasselbe Resultat findet man aus dem zweiten Schema, wenn mail bedenkt, dafs in diesem Falle die Zeichenreihe [a] mit den Zeichen - $0+$ schliefsen mufs, wenn $a$ die zwischen $\alpha$ und $\beta$ liegende Wurzel der Gleichung $f^{\prime} x=0$ ausdrückt. Man hat daher folgende Regel. Soll die Gleichung $f x=0$ unter den erwähnten Umständen zwei reelle Wurzeln haben, die zwischen $\alpha$ und $\beta$ liegen, so mufs die Summe der Quotienten $\frac{f \beta}{f^{\prime} \beta}, \frac{f \alpha}{-f^{\prime} \alpha}$ kleiner sein als der Unterschied der Grenzen; im entgegengesetzten Falle kann man mit Sicherheit annehmen, dafs zwischen den erwähuten Grenzen keine Wurzel liegt. Ist aber diese Summe wirklich kleiner als $\beta-\alpha$, so folgt daraus noch nicht, dafs die zwei reellen $W$ urzeln wirklich vorhanden sind, sondern man sieht daraus nur, dafs die Grenzen nicht eng genug gezogen sind. In diesem Falle substituire man statt $x$ eine zwischen $\alpha$ und $\beta$ liegende Zahl $c$. Hat $f c$ nicht dasselbe Zeichen wie $f \alpha$ und $f \beta$, so folgt daraus, dals eine reelle Wurzel zwischen $\alpha$ und $c$, die andere zwischen $c$ und $\beta$ liegt. Hat aber $f c$ dasselbe Zeichen, so sehe man ob $f^{\prime} c$ in Absicht auf das Zeichen mit $f^{\prime} \alpha$ oder $f^{\prime} \beta$ übereinstimmt. Man nenne $\partial$ diejenige der Zahlen $\alpha$ und $\beta$, die, in $f^{\prime} x$ substituirt, ein Resultat giebt, dessen Zeichen dem von $f^{\prime} c$ entgegengesetzt ist. Die zwei möglicherweise vorhaudenen Wurzeln müssen also zwischen $c$ und $\partial$ liegen. Man wende daher auf diese Grenzen dasselbe Verfahren an, weIches früher bei den Grenzen $\alpha$ und $\beta$ angewandt wurde. Fährt man auf diese Weise fort, so findet man zuletzt, entweder dafs keine reelle $W$ urzel zwischen den Gronzen $\alpha$ und $\beta$ liegt, oder man gelangt dahin, die Wurzeln zu trennen.

Bisher ward $\boldsymbol{r}^{2} x$ als die bestimmende Function angenommen. Nähme man aber einen höheren Differentialquotienten zur bestimmenden Function, so könnte es sein, dafs der Unterschied der $\mathbf{Z}_{\text {y }}^{e}$ ischenwechsel in den Reihen $[\alpha]$ und $[\beta]$ gröfser als 2 wäre; auch könnte sich dieser Unterschied früher als bei den zwei letzten Zeichen zeigen. Da indessen schon Fourier ausführlich gezeigt hat, dafs auch in diesen Fällen dieselbe Regel hin- 
reicht, um das Vorbandensein oder die Abwesenheit der reellen Wurzeln zu erkennen, und dieselben Betrachtungen ohne irgend eine Aenderung anch für die Wurzeln einer transcendenten Gleichung gelten, die zwischen den bestimmenden Grenzen $\alpha$ und $\beta$ angedeutet werden, so halte ich es für überflïssig, diese Betrachtungen zu wiederholen.

17. Durch das Vorhergehende ist die Frage, wie man die Grenzen fuden kann, zwischen welchen die eiuzelnen reellen $W$ urzeln enthalten sind, erledigt. Es kommt blofs darauf an, dafs man den Zwischenraum von $-\infty \mathrm{zu} \infty$ in Unterabtheilungen theilt, so dafs für jede solche Unterabtheilung irgend ein Differentialquotient zwischen den Grenzen, die diese Unterabtheilung einschliefsen, dasselbe Zeichen behält; alsdann findet man, wie viele Wurzeln zwischen diesen Grenzen liegen. Dies Verfahren wird durch die folgenden Beispiele noch deutlicher werden. Ich will nur noch eine Bemerkung hier anknüpfen. Da eine algebraische Gleichung vom mten Grade inmer das Product von $m$ einfachen Factoren ist, die den $\boldsymbol{m}$ reellen oder imaginären Wurzeln entsprechen, so kann man aus dem Verluste der Zeichenwechsel, die nicht reellen Wurzeln der Gleichung entsprechen, schliefsen, dafs die Gleichung eben so viel imagiuäre Wurzeln hat, als sie solcher Zeichenwechsel verliert. Denn da die Zeicheureihe überhaupt zwischen den Grenzen $-\infty$ und $\infty$ immer in Zeichenwechsel verliert und jeder reellen Wurzel der Verlust eines Zeichenwechsels entspricht, so folgt daraus, dafs die Zeichenwechsel, welche nicht wegen reeller Wurzeln verloren geheu, eben so viele imaginäre Wurzeln andeuten. Dieser Schlurs ist aber auf die transcendenten Gleichungen im Allgemeinen nicht anwendbar. Denu, wie früher bewiesen wurde, bestehen diese Gleichungen nicht immer aus Producten einfacher Factoren, die den Wurzeln der Gleichung entsprechen. Wenn man daher findet, $\mathrm{d}_{i i} f_{s}$ zwischen den zwei bestimmenden Grenzen $\alpha$ und $\beta$ mehré Zeichenwechsel verloren gehen als reelle Wurzeln zwischen diesen Grenzen enthalten sind, so folgt daraus noch nicht, dars die Gleichung imagiuäre Wurzeln hat.

18. Sobald einmal die reellen Wurzeln getrennt sind, so geht die genauere Berechnung ihrer Werthe ohne allen Unterschied auf dieselbe Weise fort, wie bei den algebraischen Gleichungen. Wendet man die verbesserte Newtonsche Näherungsmethode an, wie man sie bei Fourier findet, so mufs man von dem in (9.) gegebenen Satze Gebrauch machen, und verfährt auf dieselbe Weise, wie Fonrier in zweiten Buche seines Werkes 
über die Gleichungen. Ich kann daher wieder nur auf dieses Werk verweisen und will blofs in der Kürze die Regeln der Berechnung zusammenstellen, damit ihre Anwendung auf die Lösung einzelner Gleichungen, die später folgen soll, deutlicher werde.

Mau zielt zuerst die Grenzen so eng zusammen, dafs nur eine Wurzel der Gleichung $f x=0$ und keine Wurzel der Gleichungen $f^{\prime \prime} x=0$, $f^{\prime} x=0$ zwischen denselben enthalten ist. Es ist immer möglich, so enge Grenzen zu ziehen, sobald die Functionen $f^{\prime \prime} x$ und $f^{\prime} x$ nicht einen gemeinschaftichen Factor mit $f x$ haben; im entgegengesetzten Falle müfste nan erst diesen Factor suchen und absondern. Sind diese Grenzen gefunden und nennt man die kleinere $\alpha$, die gröfsere $\beta$, so bemerke man, welche derselben so beschaffen ist, dafs sie, statt $x$ in $f^{2} x$ und $f x$ substituirt, diesen Functionen gleiche Zeichen giebt: diese Grenze heifse die äufsere. Man bezeichne sie durch $\partial$, so sind

$$
\alpha-\frac{f \alpha}{f^{\prime} \partial}, \quad \beta-\frac{f \beta}{f^{\prime} \delta}
$$

zwei neue Näherungswerthe von $x$, und zwar liegt der erste zwischen $\alpha$ und $x$, der zweite zwischen $x$ und $\beta$. Mit diesen neuen Näherungswerthen kann man wieder wie mit $\alpha$ und $\beta$ verfahren und daraus zwei andere Näherungswerthe herleiten, die der Wurzel noch näher liegen, und dieses Verfahren läfst sich so weit man will fortsetzen. Mau wird aber diese zwei Grenzen nicht jedesmal zu berechnen, sondern nur folgende Regel zu beobachten haben. Man ziehe zuerst die Grenzen $\alpha$ und $\beta$ so eng zusammen, dals sie nur um eine Decimal - Einheit verschiedeu sind: ihr Unterschied sei $\left(\frac{1}{10}\right)^{n}$. Man nehme alsdann diejenige der Grörsen $f^{\prime \prime} \alpha$ und $f^{\prime \prime} \beta$, deren numerischer Werth der grölste ist, und dividire ihn durch diejenige der Grölsen $2 f^{\prime} \alpha$, $2 f^{\prime} \beta$, die den kleinsten Zahlenwerth hat. Es sei $\left(\mathrm{r}_{1}^{1}\right)^{k}$ die Decimal-Einheit, welche unmiltelbar gröfser ist als dieser Quotient. Man untersuche nun ob $n$ gleich $1-k$, oder grölser als diese Zahl ist. Ist $n<1-k$, so muls man die Grenzen enger zusammenziehen, bis die Bedingung $n \overline{>} 1-k$ erfüllt ist. Man nehme alsdann, wenn $\beta$ die äufsere Grenze ist, den Quotienten $\frac{f \beta}{f^{\prime} \beta}$ und entwickele ihn bis zur $2 n+k^{\text {ten }}$ Decimalstelle einschliefslich. Die letzte Stelle des Quotienten vermehre man um eine Einheit und addire den so gefundenen Werth zur Grenze $\beta$, oder ziehe ihn davon ab, je nachdem $f \beta$ und $f^{\prime} \beta$ verschiedene oder gleiche Zeichen baben. Der so ent- 
steliende nene Näherungswerth $\beta^{\prime}$ kann gröfser oder kleiner als die Wurzel sein; was man leicht erfährt, wenn man $\beta^{\prime}$ statt $x$ in $f x$ substituirt. In jedem Falle aber ist $\beta^{\prime}$ von $x$ um eine Grölse verschieden, die weniger als $\left(\frac{1}{1} \frac{1}{2 n+k}\right.$ beträgt. Wenn man daher die letzte Decimalstelle im Werthe von $\beta^{\prime}$ um eine Einheit vermehrt oder vermindert, so findet man eine zweite Grenze, die kleiner oder grölser als die Wurzel ist, je nachdem $\beta^{\prime}$ gröfser oder kleiner als diese ist. Mit diesen neuen Grenzen verfahre mau wieder wie mit den vorhergehenden, so erhält man allmälig Resultate, die bis auf die Decimalstellen vom Range $2 n+k, 4 n+3 k, \delta n+\tau k$ u. s. w. genau sind.

\section{Beispiele.}

19. Ich werde nun, theils um das Vorhergehende zu erläutern, theils um der dritten Anforderung der Societät Genüge zu leisten, die Werthe einiger Wurzeln verschiedener transcendenter Gleichungen berechnen, und will nur noch eine allgemeine Bemerkung machen. Im Allgemeinen steht es frei, irgend einen beliebigen Differentialquotieuten als bestimmende Function zu nehmen, sobald man Grenzen kennt, zwischen welchen er beständig dasselbe Zeichen behält. In der Regel wird es aber am zweckmäfsigsten sein, die Grenzen zu suchen, zwischen welchen $f^{\prime \prime} x$ dasselbe Zeichen behält, da gewöhnlich die Differentialquotienten einer transcendenten Function desto verwickelter werden, je weiter man die Differentiation fortsetzt, es also auch schwerer sein wird, die Grenzen zu bestimmen, zwischen welchen eine solche Function dasselbe Zeichen behält. Besteht aber der erste Theil der Gleichung theils aus transcendenten, theils aus algebraischen Functionen, so wird es häufig nützlich sein, die Differentiation so weit fortzusetzen, bis die algebraischen Functionen verschwunden sind. Hätte man z. B. die Gleichung

$$
f x=\sin x+5 x^{3}-4 x^{2}+3 x-6=0,
$$

so würde man viermal zu differentiiren haben. Dies giebt $f^{4} x=\sin x$, und man nimmt alsdann $\sin x$ als bestimmende Funclion.

20. In Beziehung auf die Schwierigkeit, welche die Auflösung der transcendenten Gleichungen im Verlıälınifs zu der der algebraischen Gleichungen macht, kann man die transceudenten Gleichungen in verschiedene Oassen eintheilen. 
Am leichtesten sind offenbar diejenigen Gleichungen aufzulösen, deren einzelne Glieder nur ganze oder gebrochene Potenzen einer und derselben transcendenten Function enthalten. Solche Gleichnngen sind z.B. die folgenden:

1. $\boldsymbol{A}(\sin x)^{\alpha}+\boldsymbol{B}(\sin x)^{\beta}+\boldsymbol{C}(\sin x)^{\gamma}+\ldots M(\sin x)^{\mu}=0$, 2. $\boldsymbol{A} e^{\alpha t}+B e^{\beta t}+C e^{r t}+\ldots M e^{\mu t}=0$,

wo $\boldsymbol{A}, \boldsymbol{B}, \boldsymbol{C}, \ldots \boldsymbol{M}$ bestimmte $\boldsymbol{Z}$ ahlen und $\alpha, \beta, \gamma$. ebenfalls reelle, ganze oder gebrochene $Z$ ahlen siud. Solche Gleichungen kann man nemlich unmiltelbar auf algebraische bringen. Man braucht nur statt $\sin x$ in der ersten oder statt $e^{t}$ in der zweilen Gleichung eine neue unbekannte Grölse, etwa y. $\mathrm{zu}$ setzen, so gehen die Gleichungen in folgende Gleichung über:

3. $\boldsymbol{A} y^{\alpha}+\boldsymbol{B} y^{\beta}+\boldsymbol{C} y^{\gamma}+\ldots M y^{\mu}=0$.

Dasselbe ist der Fall bei der Gleichung

4. $\boldsymbol{A} \boldsymbol{a}^{x}+\boldsymbol{B} \boldsymbol{b}^{x}+\boldsymbol{C} \boldsymbol{c}^{x}+\ldots+\boldsymbol{M} \boldsymbol{m}^{x}=0$,

wo $A, B, C, \ldots$ beliebige Zahlen und $a, b, c, \ldots$ positive Zahlen sind. Man braucht nur $a=e^{a}, b=e^{i}, c=e^{\gamma} \ldots$ zu setzen, so geht die Gleichung (4.) in die Gleichung (2.) über. Sind die Exponenten $\alpha, \beta, \gamma, \ldots$ in der Gleichung (3.) ganze positive Zahlen, so ist die Gleichung eine gewölnliche algebraische. Sind aber einige dieser Exponenten negative oder gebrochene Zahlen, so hat man nicht nöthig, wie es gewöhnlich in solchen Fällen geschieht, diese Exponenten durch Potenziiren wegzuschaffen, sondern man kann sie unmittelbar nach denselben Regeln behandeln, welche Fourier für die gewöhnlichen algebraischen Gleichungen gegeben hat. Da hierüber Sturm schon eine Abhandlung geschrieben hat, die vielleicht bereits gedruckt ist, so werde ich mich nicht länger bei diesem Gegenstande auflualten und verweise auf dessen Bemerkungen *).

21. Znnächst lasse ich die transcendenten Gleichungen folgen, bei welchen sich zwar der erste 'Theil der Gleichung selbst nicht auf eine algebraische Function zurückführen läfst, aber die sämmtlichen Differentialquotienten algebraische Functionen sind. Dies ist der Fall, wenn in der Gleichung die Functionen $\log x, \arcsin x, \arccos x, \operatorname{arctang} x$ etc. vorkommen. Eine solche Gleichung ist z. B. $x^{x}-100$,

$$
x \log x-1+f_{0}=0 \text {, }
$$

welche Euler behandelt bat $\left.{ }^{*} *^{*}\right)$. Hier ist, wenn man die natürlichen Loga-

*) Bulletin des sciences par Ferussac, Sect. I. T. II. 1829.

*) Instit. calc. différ. Tom. II. \$. 243.

Crelle's Journal d. M. Bd. XXII. Hft. 1. 
rithmen anwendet,

$$
\begin{aligned}
f x & =x \log x-10 \\
f^{\prime} x & =\log x+1 \\
f^{2} x & =\frac{1}{x}
\end{aligned}
$$

Da $f^{2} x$ zwischen den Grenzen $x=0$ und $x=\infty$ positiv ist, so nehme man diese Function zwischen diesen Grenzen als die besiminende an. Da $x$ und $\log x$ fortwälrend wachsen, so folgt, dafs zwischen diesen Grenzen nur eine Wurzel der Gleichung $f x=0$ liegen kann. Setzt man $x=3$, und dann $x=4$, so findet man

$$
\begin{aligned}
& {[3]=+\underset{\frac{1}{3}}{+} \underset{2,098 \ldots}{+} \ldots .} \\
& {[4]=\underset{\frac{1}{4}}{+} \underset{2,386}{+} \ldots \quad \stackrel{0,940 \ldots .}{t}}
\end{aligned}
$$

Es liegt also die Wurzel zwischen 3 und 4. Nun ist $4-3=\left(\frac{1}{x_{0}^{1}}\right)^{0}$ und $\frac{t}{2.2,098}=0,07$, also (nach \$. 18.)

$$
k=1, \quad n=0,
$$

mithin $n=1-k$. Die Grenzen sind also eng genug zur nähernden Berechnung gezogen. Die äufsere Grenze ist hier 4 , mithin $\frac{f \beta}{f^{\prime} \beta}=\frac{0,940}{2,386}$, und da $2 n+k=1$ ist, so mufs die Division bis zur ersten Decimalstelle ausgeführt werden: also ist $\frac{f \beta}{f^{\prime} \beta}=0,3$ und mithin der erste Nïherungswerth $=4-0,4=3,6$.

Substituirt man in $f x$ statt $x$ den Werth 3,6, so erhält man ein positives Resultat. Der Werth 3,6 ist also zu grofs, und die Wurzel liegt zwischen 3,5 und 3,6. Nun ist $n=1$, also $2 n+k=3$. Da 3,6 die äufsere Grenze ist, so hat $\operatorname{man} \frac{f(3,6)}{f^{t}(3,6)}=\frac{0,00619 \ldots .}{2,28093 \ldots .}=0,002$; also ist der zweite Näherungswerth $3,6-0,003=3,597$ bis auf $\left(\mathrm{T}^{\frac{1}{0}}\right)^{3}$ genau. Da $f(3,597)$ negativ ist, so liegt die Wurzel zwischen 3,597 und 3,598, und da 3,598 die äufsere Grenze ist, so hat man $\frac{f(3,598)}{f^{\prime}(3,598)}=\frac{0,00163032}{2,28037813}$, welcher Quotient bis zur 7ten Decimalstelle entwickelt werden mufs, indem $2 n+k=7$ ist. Dies giebt 0,0007149; also ist der dritte Näherungswerth 3,598-0,000715 $=3,597285$ bis auf $\left(\frac{1}{1} \delta\right)^{7}$ genau, und zwar liegt der Werth zwischen 3,5972850 und 3,597285,1. Euler findet 3,5972852. 
22. Ich werde nun einige Gleichungen abhandeln,' für welche die Differentialquotienten ebenfalls transcendente Grölsen sind, jedoch vou der Art, dafs die numerischen Werthe der darin vorkommenden transcendenten Functionen bereits in Tafeln berechnet sind. Hierher gehören zunächst die trigonometrischen Functionen. Es sei also z. B. die Gleichung

$$
f x=x-\cos x=0
$$

gegeben, welche Euler ebenfalls behandelt hat *). Es ist einleuchtend, dafs diese Gleichung nur eine reelle Wurzel haben kann. Nun ist

$$
\begin{aligned}
& f^{\prime} x=1+\sin x, \\
& f^{\prime \prime} x=\cos x .
\end{aligned}
$$

Da $\cos x$ zwischen den Grenzen $x=0, x=90^{\circ}$ immer positiv ist, so kann $f^{\prime \prime} x$ zwischen diesen Grenzen als bestimmende Function angenommen werden; $x-\cos x$ ist aber negativ, wenu man $x=0$ setzt, und positiv, wenn mau $x=90^{\circ}$ setzt, woraus schon folgt, dafs eine Wurzel der Gleichung $x-\cos x=0$ zwischen diesen Grenzen enthalten ist. Zieht man die Grenzen enger zusammen und nimmt als solche zuerst $x=0,7, x=0,8$, so findet man

$$
\begin{aligned}
& {[0,7]=\underset{0,764 \ldots . .1,644 \ldots . .0,064 \ldots .}{+}} \\
& {[0,8]=\underset{0,696 \ldots}{+} \underset{1,717 \ldots .}{+} \underset{0,103 \ldots}{+}}
\end{aligned}
$$

Hier ist $\frac{0,764}{2.1,644}=0,2$ also $k=0$; auch ist $m=1$; mithin sind die Grenzen eng genug zur nähernden Berechnung. Die äufsere Grenze ist $\boldsymbol{x}=0,8$, und da $2 n+k=2$ ist, so entwickele man $\frac{f(0,8)}{f^{\prime}(0,8)}=\frac{0,103}{1,717}$ bis zur zweiten Decimalstelle. Dies giebt 0,06: also ist der erste Näherungswerth $=0,8-0,07=0,73$ bis auf $\left(\frac{1}{10}\right)^{2}$ genau. Substituirt man diesen Werth statt $x$, so findet man, dafs er zu klein ist; der Werth von $x$ liegt also zwischen 0,73 und 0,74 und man hat $\frac{f(7,4)}{f^{\prime}(7,4)}=\frac{0,001531}{1,674 \ldots .}$, welcher Quotient bis auf die vierte Decimalstelle entwickelt werden murs. Dies giebt 0,0009; also ist der zweite Näherungswerth $0,74-0,001=0,739$ bis auf $\left(\frac{1}{10}\right)^{4}$ genau. Durch Substitution dieses Werthes statt $x$ findet man, dafs die Wurzel zwischen 0,739 und 0,7391 liegt. Der nächste Quotient mufs bis auf die 8te Decimalstelle berechnet werden. Dies giebt $\frac{f(0,7391)}{f^{\prime}(0,7391)}=$

*) Introd. in anal. infinit. L. II. \$. 531. 
$\frac{0,000024887 \ldots \ldots}{1,67362 \ldots \ldots}=0,00001487$; also ist der dritte Näherungswerth 0,7391 $-0,00001488=0,73908512$ bis auf $\left(\frac{1}{10}\right)^{8}$ genau. Euler findet $0,7390847$.

23. Es : sei ferner die Gleichung

$$
x-\operatorname{tang} x=0
$$

gegeben, welche in der Theorie der Schwingungen elastischer Körper and in der Theorie der Wärme vorkommt. Statt dieser Gleichung kann man auch schreiben $\frac{x \cos x-\sin x}{\cos x}=0$, und man kann hier wieder wie früher beweisen, dafs die Wurzeln der Gleichung $\frac{1}{\cos x}=0$ nicht Wurzeln der gegebenen Gleichung sind. (Vergl. \$. 5.) Man braucht daher uur die Gleichung $x \cos x-\sin x=0$ zu betrachten. Da diese Gleichung unverändert - $y_{x}$ bleibt, wenn man $-x$ statt $x$ setzt, so folgt, dals jeder positiven Wurzel $a$ n eine negative - $\alpha$ entspricht. Man braucht daher nur die 'positiven Wurzelı zu suchen. Es ist

$$
\begin{aligned}
f x & =x \cos x-\sin x, \\
f^{\prime} x & =-x \sin x, \\
f^{\prime \prime} x & =-(x \cos x+\sin x) .
\end{aligned}
$$
nes, so ist klar, dals $f^{\prime \prime} x$ zwischen den Grenzen $x=\omega$ und $x=90$ immer negativ ist. Man hat also

$$
\begin{aligned}
& {[\omega]=--} \\
& {\left[90^{\circ}\right]=--}
\end{aligned}
$$

$\mathbf{Z}$ wischen diesen Grenzen liegt mithin keine Wurzel. Es ist ferner einleuchtend, dafs keine Wurzel zwisehen den Grenzen $x=90^{\circ}$ und $x=180^{\prime \prime}$ enthalten ist, da $\cos x$ zwischen diesen Grenzen inmer negativ und $\sin x$ immer positiv ist. Zwischen den Grenzen $x=180^{\circ}$ und $x=270^{\circ}$ ist $f^{\prime \prime} x$ immer positiv, und kann daher zwischen diesen Grenzen als bestimmende Function angenommen werden. Nun findet man

$$
\begin{aligned}
{\left[180^{\prime}+\omega\right] } & =++- \\
{\left[270^{\circ}\right] } & =+++;
\end{aligned}
$$

also hat die Zeichenreihe $[180+\omega]$ einen Zeichenwechsel mehr als die Zeichenreihe [270'], und es liegt daher eine Wurzel zwischen diesen Grenzen. Zieht man die Grenzen enger zusammen, so ergiebt sich, dals die Wurzel zwischen $x=4,4$ und $x=4,5$ liegt, und zwar ist 
1. Stern, ̈̈ber die Auflösung der transcendenten Gleichungen.

$$
\begin{aligned}
& {[4,4]=\underset{2,3}{+} \underset{4,187 \ldots .}{+} \quad-\quad 0,4006 \ldots .} \\
& {[4,5]=\underset{1,92}{+} \underset{4,398885}{+} \underset{0,028949}{+}}
\end{aligned}
$$

Nun ist $\frac{2,3}{8,37}=0,3$, also $k=0$; ferner ist $n=1$; mithin sind die Grenzen eng genug zur nähernden Berechnung. Die äufsere Grenze ist 4,5. Man mufs also den Quotienten $\frac{0,028 \ldots .}{4,398 \ldots}$ bis auf die zweite Decimalstelle entwickeln. Dies giebt 0,00. Mithin ist der erste Näherungswerth 4,5-0,01 $=4,49$. Da $f(4,49)$ negativ ist, so folgt hieraus, dafs die Wurzel zwischen 4,49 und 4,5 enthalten ist. Nun ist 4,5 die äufsere Grenze, und der Quotient $\frac{0,028949}{4,398885}$, auf vier Decimalstellen entwickelt, giebt 0,0065 ; also ist der zweite Näherungswerth 4,5-0,0066 = 4,4934. Dieser Werth ist zu klein; also liegt die Wurzel zwischen 4,4934 und 4,4935. Der Quotient $\frac{f(4,4935)}{f^{\prime}(4,4935)}=\frac{0,000396339}{4,38627}$. auf 8 Decimalstellen entwickelt, giebt 0,00009035: also ist der dritte Näherungswerth $4,4935-0,00009036=4,49340964$ bis auf $\left(\frac{1}{10}\right)^{8}$ genau. In Bogen-Einheiten ausgedrückt, giebt dieses $257^{\prime} 27^{\prime} 12^{\prime \prime}, 268$. Euler findet den Näherungswerth $\left.25^{\prime \prime} 27^{\prime} 12^{\prime \prime}=4,49340837^{\prime \prime}\right)$. Man kann aber vermittelst der Eulerschen Methode den Werth noch genauer finden, wem man alle Glieder der Reihe, die er zur Berechnung anwendet und welehe noch auf die achte Decimalstelle Einflufs haben, berücksichtigt. Man findet alsdann, nahe mit unserem Resultate zusammentreffend, den Werth 4,49340 68. Poisson findet **) 4,49331, welcher Werth schon in der vierten Decimalstelle ungenau ist und wahrscheinlich 4,49341 heifsen soll.

Auf dieselbe Weise, wie hier die kleinste positive Wurzel gefunden wurde, können nun auch die übrigen Wurzeln berechnet werden. Es ist aber einleuchtend, dafs es unendlich viele solche Wurzeln giebt und dafs die $n$ te Wurzel zwischen den Grenzen $n \pi$ und $\left(n+\frac{1}{2}\right) \pi$ enthalten ist, wo $\pi$ die halbe Peripherie bedeutet.

Bemerkenswerth ist auch die Art, wie Euler durch Umkehrung der Reihen Näherungswerthe für jede nte Wurzel findet, die desto genauer sind,

*) Introd. in anal. infinit. L. II. \$. 539.

* Mém. de l'acad. des sciences T. VII. p. 420. Als Werth der zweiten Wurzel giebt Poisson 7,73747, was schon in der zweiten Decimalstelle unrichtig ist, indem der wahre Werth $7,725 \ldots$ ist. 
je gröfser $\boldsymbol{n}$ ist. Die Brauchbarkeit dieses Verfahrens in diesem besondern Falle bängt aber von dem besondern Umstande ab, dafs sich die nte Wurzel dem Werthe $\left(n+\frac{1}{2} \pi\right)$ immer mebr nähert, je grölser $n$ ist (vergl. \$. 2.).

24. Eine andere Gleichung dieser Art, welche in der Theorie der Schwiugungen einer elastischen Kugel vorkommt, ist folgende:

$$
\left(4-3 x^{2}\right) \sin x-4 x \cos x=0 \text {. }
$$

Auch hier entsprieht jeder posiliven Wurzel $\alpha$ eine negative - $\alpha$; man braucht daher nur die positiven Wurzeln zu suchen. Die kleinste entspricht dem Werthe $x=0$, Die nächste Wurzel findet man wie folgt. Es ist

$$
\begin{aligned}
f x & =\left(4-3 x^{2}\right) \sin x-4 x \cos x, \\
f^{\prime} x & =-x(3 x \cos x+2 \sin x), \\
f^{\prime \prime} x & =\left(3 x^{2}-2\right) \sin x-8 x \cos x .
\end{aligned}
$$

Die Function $f^{\prime \prime} x$ ist zwischen den Grenzen $x=0$ und $x=45^{\circ}$ immer negativ. Denn ist $3 x^{2}<2$ oder $x<\sqrt{\frac{2}{3}}$, das heirst $x<45^{\prime}$, so ist $3 x^{2}-2$ und also auch $f^{\prime \prime} x$ negativ: mithin kann $f^{\prime \prime} x$ zwischen diesen Grenzen zur bestimmenden Function genommen werden. Bezeichnet man durch $\omega$ ein Unendlich-Kleines, so hat man

$$
\begin{aligned}
{[\omega] } & =- \\
{\left[45^{\circ}\right] } & =-
\end{aligned}
$$

Es liegt also keine Wurzel zwischen 0 und $45^{\circ}$. Zwischen den Grenzen $x=45^{\circ}$ und $x=90^{\circ}$ kann aber $f^{\prime \prime} x$ nicht als bestimmende Function gebraucht werden, weil sie zwischen diesen Grenzen das Zeichen wechselt. Man könnte nun zwar den Zwischenraum in kleinere theilen, wird aber schneller zum Ziele kommen, wenn man die höhern Differentialquotienten entwickelt. Denn es ist

$$
\begin{aligned}
& f^{\prime \prime \prime} x=\left(3 x^{2}-10\right) \cos x+14 x \sin x \\
& f^{\prime r} x=\left(24-3 x^{2}\right) \sin x+20 x \cos x .
\end{aligned}
$$

Offenbar ist $f^{\mathrm{t}} x$ zwischen den Grenzen 0 und $90^{\prime \prime}$ immer positiv und folglich zwischen diesen Grenzen als bestimmende Function zu gebrauchen. Die Zeichenreihen sind

$$
\begin{aligned}
{[\omega] } & =+--- \\
{\left[90^{\prime}\right] } & =+++
\end{aligned}
$$

Da nun jede Zeichenreihe nur einen Zeichenwechsel enthält, so liegt keine Wurzel zwischen diesen Grenzen.

Zwischen den Grenzen $x=90^{\circ}$ und $x=180^{\prime \prime}$ ist $f^{\prime \prime} x$ immer positiv und daher zwischen diesen Grenzen als bestimmende Function brauchbar. 
Man findet

$$
\begin{gathered}
{\left[90^{\circ}\right]=+-\overline{ }} \\
{\left[180^{\circ}\right]=++}
\end{gathered}
$$

also ist zwischen diesen Grenzen eine Wurzel enthalten. Die Grenzen sind aber noch nicht eng genug zur nähernden Berechnung, da auch $f^{\prime} x=0$ eine Wurzel zwischen diesen Grenzen hat. Zieht man die Grenzen enger zusammen und setzt allenfalls $x=2,5$ und $x=2,6$, so hat man

$$
\begin{aligned}
& {[2,5]=\underset{26,04}{+} \underset{12,029}{+} \quad 0,816 \ldots .} \\
& {[2,6]=\underset{27,2}{+} \underset{14,707}{+} \underset{0,519}{+} \ldots}
\end{aligned}
$$

Hier ist $\frac{27,2}{2.12,029}=1, \ldots$, also $k=-1$; auch ist $n=1$; mithin wird die Bedingung $n>1-k$ nicht erfült und die Grenzen müssen enger gezogen werden. Setzt man $x=2,56, x=2,57$, so findet man

$$
\begin{aligned}
& {[2,56]=\underset{26,81}{+} \underset{13,901}{+} \ldots} \\
& {[2,57]=\underset{26,92}{+} \underset{13,88437}{+} \underset{0,09057 \ldots .}{+}}
\end{aligned}
$$

Hier ist $\frac{26,92}{2.13,8}=0,9$, also $k=0$; ferner $n=2$; folglich sind die Grenzen hiulänglich nahe. Die äufsere Grenze ist 2,57; der Quotient $\frac{0,09057}{13,88437}$ auf vier Stellen entwickelt, giebt 0,0065: also ist der erste Näherungswerth $2,75-0,0066=2,5634$. Dieser Werth ist zu klein; die Wurzel liegt also zwischen 2,5634 und 2,5635. Bei der nächsten Operation erhält man den Werth bis auf 8 Stellen genau, und zwar ist

$$
\frac{f(2,5635)}{f^{\prime}(2,5635)}=\frac{0,00090157}{13,7095659}=0,00006576,
$$

also der zweite Näherungswerth $2,5635-0,00006577=2,56343423$. Poisson findet 2,56334 *).

Zwischen den Grenzen $x=180^{\circ}$ und $x=270^{\circ}$ ist $f x$ immer, positiv, mithin keine Wurzel zwischen diesen Grenzen enthalten. Im vierten Quadranten dagegen liegt eine Wurzel; denn $f^{\prime \prime} x$ ist zwischen diesen Grenzen immer negativ. Man kann also diese Function wieder als bestimmende ansehen und findet

$$
\begin{aligned}
& {\left[270^{\circ}\right]=-++} \\
& {\left[360^{\circ}\right]=---}
\end{aligned}
$$

*) Mémoires de Yacad. des se. T. VUI. p. 420. 
Die Zeichenreihe $\left[360^{\circ}\right]$ hat also einen Zeichenwechsel weniger als die Zeichenreihe [270" . Zieht man die Grenzen enger zusammen, so findet man

$$
\begin{aligned}
& {[6,0]=\overline{75,7} \quad \overline{100,34} \underset{6,01}{+}} \\
& {[6,1]=\overline{67,95} \overline{107,53} \overline{4,38}}
\end{aligned}
$$

Hier ist $\frac{75,7}{2.100,34}=0,3$, also $k=0, n=1$ und $n=1-k$.

Da 6,1 die äufsere Grenze ist, so entwickele man den Quotienten $\frac{4,38}{107,53}$ bis auf die zweite Decimalstelle. Dies giebt 0,04; also ist der erste Nïherungsuerth $6,1-0,05=6,05$. Da $f(6,05)$ negativ ist, so liegt die $\mathbf{W u r -}$ zel zwischen 6,05 und 6,06 . Nun ist $\frac{f(6,06)}{f^{\prime}(6,06)}=\frac{0,1393}{104,75}=0,0013$ : also ist der zweite Näherungswerth 6,06-0,0014 $=6,0586$. Dieser Werth ist zu klein und daher die Wurzel zwischen 6,0586 und 6,0587 enthalten. Nun ist $\frac{f(6,0587)}{f^{\prime}(6,0587)}=\frac{0,0031285}{104,6627}=0,00002989$; also ist der dritte Näherungswerth $6,0587-0,0000299=6,0586 \% 01$ bis auf $\left({ }_{1}^{1} 0\right)^{8}$ genau. Poisson hat 6,05973 .

Es ist einleuchtend, dafs auch diese Gleichung unendlich viele reelle Wurzeln hat und dafs die nte Wurzel zwischen $\left(n-\frac{1}{2}\right) \pi$ und $n \pi$ enthalten ist. Jede dieser Wurzeln kann nach den vorhergehenden Verfahren bis auf jede beliebige Decimalstelle geuau gefunden werden. Wo es sich aber nur um eine ungefähre Annäherung handelt, kann man auch hier mit Nutzen das bei dem früheren Beispiele erwähute Eulersche Verfahren anwenden und durch Umkehrung der Reilen eine Formel finden, welche die folgenden W urzelı desto genauer giebt, je grörser $\boldsymbol{n}$ ist.

26. Ich werde nun einige Aufgaben behandeln, bei welchen aufser den trigonometrischen Functionen auch die Fuuctionen $e^{x}+e^{-x}$ und $e^{x}-e^{-x}$ vorkommen. Auch zur Berechnung dieser Functionen besitzen wir jetzt Tafeln in der vortrefflichen Abhandlung Gudermanns über die Theorie der Polenzialfunctionen $*$. Ich werde nach dem Vorgange dieses Gelehrten zur Alkürzung $\frac{1}{2}\left(e^{x}+e^{-x}\right)=\operatorname{Cos} x$ und $\frac{1}{2}\left(e^{x}-e^{-x}\right)=\operatorname{Sin} x$ selzen.

Es sei nun die Gleichung

$$
f x=\left(e^{x}+e^{-x}\right) \cos x-2=0
$$

*) Crelle, Journal für die Mathematik, Bd. 6. u. 7. 
gegeben, statt welcher man auch

$$
\operatorname{Cos} x \cos x-1=0
$$

setzen kann. Es ist klar, dafs jeder positiven Wurzel $x$ eine negative $-x$ und eine imaginäre $\pm x \sqrt{ }-1$ entsprieht.

Hier hat man nun

$$
\begin{aligned}
f x & =\operatorname{Cos} x \cos x-1 \\
f^{\prime} x & =\cos x \operatorname{Sin} x-\sin x \operatorname{Cos} x, \\
f^{\prime \prime} x & =-2 \sin x \operatorname{Sin} x .
\end{aligned}
$$

Die kleinste positive Wurzel der Gleichung ist $x=0$. Bezeichnet alsdann $\omega$ ein Unendlieh-Kleines, so ist $f^{\prime \prime} x$ zwischen den Grenzen $x=\omega$ und $x=90^{\circ}$ immer negativ und kann daher zwischen diesen Grenzen als bestimmende Function gebraucht werden. Es ist aber

$$
\begin{gathered}
{[\omega]=--} \\
{\left[90^{\circ}\right]=--\longrightarrow}
\end{gathered}
$$

also liegt keine Wurzel zwischen diesen Grenzen. Dafs zwischen $x=90^{\circ}$ und $x=270^{\prime \prime}$ keine Wurzel liegt, ist von selbst klar, da $f x$ zwischen diesen Grenzen immer negativ ist. Zwischen den Grenzen $x=2 \% 0^{\circ}$ und $x=360^{\circ}$ ist $f^{\prime \prime} x$ immer positiv und kann daher wieder als bestimmende Function gebraucht werden. Nun ist

$$
\begin{aligned}
{[2 \%} & =++- \\
{\left[360^{\prime \prime}-\omega\right] } & =+++;
\end{aligned}
$$

also ist eine Wurzel zwischen diesen Grenzen enthalten. Zieht man die Grenzen enger zusammen und setzt zuerst $x=4,7$ und dann $x=4,8$, so ergiebt sich

$$
\begin{aligned}
& {[4,7]=\underset{109,8}{+}+\frac{}{54,28}} \\
& {[4,8]=\underset{118,2}{+}+\underset{65,84}{+}+}
\end{aligned}
$$

Ls ist aber $\frac{118,2}{2.54,2}=1, \ldots$, also $k=-1, n=1$, das heifst, die Grenzen sind nicht eng genug zur nähernden Berechnung. Setzt man ferner $x=4,73, x=4,74$, so ergiebt sich

$$
\begin{aligned}
& {[4,73]=\underset{113,26}{+} \underset{57,6409}{+}-{ }_{0,0023}} \\
& {[4,74]=\underset{114,38}{+} \underset{58,7791}{+} \underset{0,5796}{+}}
\end{aligned}
$$

Hier ist $\frac{114,3}{2 \cdot 57,6}=0,9$, also $k=0, n=2$.

Crelle's Journal d. M. Bd. XXII. IIft. 1. 
Die āufsere Grenze ist 4,74 und der Quotient $\frac{f(4,74)}{f^{\prime}(4,74)}=\frac{0,5796}{58,7791}$ muls auf vier Decinalstellen entwickelt werden. Dies giebt 0,0098 ; also ist der erste Näherungswerth 4,74-0,0099 $=3,7301$. Dieser Werth ist zu grofs, mithin die Wurzel zwischen 4,7300 und 4,7301 enthalten. Der Quotient $\frac{f(4,7301)}{f^{\prime}(4,7301)}$ auf 8 Decimalstellen entwickelt, giebt $\frac{0,00340159}{57,6512988}=0,00005900$; also ist der zweite Näherungswerth 4,7301-0,00005901 = 4,73004099.

Die hier abgehandelte Gleichung kommt bekanntlich in der Theorie der Schwingungen elastischer Stäbe vor. Statt des hier gefundenen Werthes der ersten Wurzel hat Poisson 4,\%3003 *

26. Auch die Gleichung

statt welcher man

$$
\left(e^{x}+e^{-x}\right) \cos x+2=0 \text {, }
$$

$$
\cos x \operatorname{Cos} x+1=0
$$

schreiben kanu, komint in der Theorie der Schwingungen elastischer Stäbe vor. Hier ist

$$
\begin{aligned}
f x & =\cos x \operatorname{Cos} x+1 \\
f^{\prime} x & =\cos x \operatorname{Sin} x-\sin x \operatorname{Cos} x \\
f^{\prime \prime} x & =-2 \sin x \operatorname{Sin} x
\end{aligned}
$$

Zwischen $x=0$ und $x=90^{\prime \prime}$ ist $f x$ inmer positiv; es kann also keine Wurzel zwischen diesen Grenzen liegen. Zwischen den Grenzen $x=90^{\circ}$ und $x=180^{\circ}$ ist $f^{\prime \prime} x$ immer negativ und kann daher als bestimmende Function gebraucht werden. Man hat

$$
\begin{gathered}
{\left[90^{\circ}\right]=--+} \\
{\left[180^{\circ}\right]=---;}
\end{gathered}
$$

es liegt also eine Wurzel zwischen diesen Grenzen. Nimmt man nun die engeren Grenzen $x=1,8$ und $x=1,9$, so findet man

$$
\begin{aligned}
& {[1,8]=-\overline{5,7} \quad-\frac{+}{3,69} \quad+} \\
& {[1,9]=-\underset{6,11}{4,29}-}
\end{aligned}
$$

Hier ist $\frac{6,1}{2.3,69}=0,8$, also $k=0, n=1$; der Quotient $\frac{0,10}{4,29}$ mruls da-

*) Mém. de l'acad. des sc. T. VIII. p. 485. In dem Traité de mécanique ed. 2. T. 2. p. 389 dagegen steht der Werth 4,74503; was aber offenbar 4,73003 heilsen mufs, da dieser Werth $=\frac{8}{2} \pi+0,01765$ sein soll. 
her auf zwei Decimalstellen entwickelt werden. Dies giebt 0,02; also ist der erste Näherungswerth $1,9-0,03=1,87$. Nun ist $f(1,87)$ positiv; folglich mufs die Wurzel zwischen 1,87 und 1,88 liegen. Nan hat $\frac{f(1,88)}{f^{\prime}(1,88)}=\frac{0,02033}{4,16792}=0,0048$; also ist der zueite Näherungswerth $1,88-0,0049=1,8751$. Dieser Werth ist zu klein; mithin liegt die Wurzel zwischen 1,8751 und 1,8752. Der folgende Näheruugswerth kann bis auf 8 Stellen genau gefunden werden, und zwar findet man $\frac{f(1,8752)}{f^{\prime}(1,8752)}=\frac{0,00039722}{4,138717}=0,00009597$; also ist der dritte Nüherungswerth 18752-0,00009598 =1,87510402. Poisson findet in der erwähnten Abhandlung $1,8756 *$ *).

27. Ieh will nun noch einige Gleichungen abhandeln, bei welchen der Werth von $f x$ in einer convergirenden Reihe ausgedrückt ist, für welche noch keine Tafeln berechnet sind. Ich nehme zuerst die Gleichung

1. $r x=1-x+\frac{x^{2}}{(1.2)^{2}}-\frac{x^{3}}{(1.2 .3)^{2}}+\frac{x^{4}}{(1.2 .3 .4)^{2}}-\ldots=0$, welche bekanntlich in der Theorie der Bewegung der Wärme in einem Cylinder und bei mehreren anderen physikalisch - mathematischen Untersuchungen vorkommt **. Diese Gleichung kann keine negativen Wurzeln haben, da alle Glieder der Reihe positiv werden, sobald man statt $x$ eine negative Zabl setzt. Man braucht also nur die positiven Wurzeln zu suchen. Hier ist

$$
\begin{aligned}
& \text { 2. } f^{\prime} x=-1+\frac{x}{2}-\frac{x^{2}}{2^{2} \cdot 3}+\frac{x^{3}}{2^{2} \cdot 3^{2} \cdot 4}-\frac{x^{4}}{2^{2} \cdot 3^{2} \cdot 4^{2} \cdot 5}+\ldots \\
& \text { 3. } f^{\prime \prime} x=\frac{1}{2}-\frac{x}{2.3}+\frac{x^{2}}{2^{2} \cdot 3.4}-\frac{x^{3}}{2^{2} \cdot 3^{2} \cdot 4.5}+\ldots
\end{aligned}
$$

Die drei Reihen (1., 2., 3.) sind nach bekannten Sätzen convergirende Reihen, und es kann daher vermittelst ihrer der Werth von $f x, f^{\prime} x, f^{\prime \prime} x$ für jedes gegebene $x$ mit jedem beliebigen Grade von Genauigkeit berechnet werden, sobald $x$ eine endliche, reelle Grölse ist. Bei diesen Reihen kommt man nemlich, wenn man statt $x$ irgend einen beliebigen endlichen reellen Werth setzt, jedesmal an ein Glied, von welchem an gerechnet der Zahlenwerth

*) Der Werth 1,87011, den Poisson im Traité de méc. T. II. p. 390 giebt, ist offenbar unrichtig und muls 1,87511 heifsen, da dieser Werth $=\frac{1}{2} \pi+\delta^{\prime}$ sein soll, wo $\delta^{\prime}=0,30431$ ist.

") Auch diese Gleichung behandelt Poisson in der erwähnten Abhandlung p. 522. 
jedes Gliedes gröfser ist als der des nachfolgenden. Bei der Reihe (1.) z. B. erhält man aus jedem Gliede $\frac{x^{m}}{2^{2} \cdot 3^{2} \ldots m^{2}}$, wenn man es mit $\frac{x}{(m+1)^{2}}$ multiplicirt, das rachfolgende. Sobald also $m$ so grofs ist, dafs $x<(m+1)^{2}$ ist, so wird jedes folgende Glied kleiner als das vorhergehende und die Glieder convergiren zu dem Werthe Null hin. Bezeichnet man daher im Allgemeinen diese Reihe durch

$$
f(x)=a_{0}-a_{1}+a_{2}-a_{3}+\ldots .+a_{m}-a_{m+1}+a_{m+2}-a_{m+3} \ldots .,
$$

setzt $a_{0}-a_{1}+a_{2}-\ldots .-a_{m \rightarrow 1}=S$ und nimmt an, dafs von $a_{m}$ an jedes Glied gröfser ist als das folgende, so hat mau

$$
f x>S, \quad f x<S+a_{m}, \quad f x>S+a_{m}-a_{m+1} \text { elc. }
$$

so dafs man also, indem man die einzelnen Glieder der Reihe zusammen addirt, sich nicht blofs dem wahren Werthe immer mehr nähert, sondern anch fortwährend zwei Grenzen hat, zwischen welchen der Werth der Reihe eingeschlossen ist. Die ersten Ziffern, welche den beiden Grenzen gemeinscbaftlich sind, müssen daher auch nothwendig dem wahren Werthe der Reihe angehören, und man kann mithin diesen $W$ erth bis auf jede beliebige Stelle genau berechnen. Aelunliches gilt auch von den übrigen Reihen.

In der Reihe $f^{\prime \prime} x$ werden zwei auf einander folgende Glie(ler allgemein durch

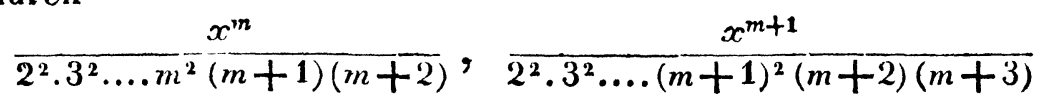

ausgedrückt. Soll nun $\frac{x^{m}}{2^{2} \cdot 3^{2} \ldots . m^{2}(m+1)(m+2)}>\frac{x^{m+1}}{2^{3} \cdot 3^{2} \ldots .(m+1)^{2}(m} \overline{+2)(m+3)}$ sein, so mufs $1>\frac{x}{(m+1)(m+3)}$ oder $x<(m+1)(m+3)$ sein. Selzt man $m=2$, so ist $(m+1)(m+3)=15$ : sobald also $x$ kleiner als 15 ist, ist jedes positive Glied der Reihe $f^{\prime \prime} x$, von $\frac{x^{2}}{2^{2} .3 .4}$ an gerechnet, gröfser als das nachfolgende negative: so lange aber $x$ nicht gröfser als 3 ist, bleibt auch $\frac{1}{2}-\frac{x}{2.3}$ positiv. Die Function $f^{\prime \prime} x$ ist daher zwischen den Grenzen $x=0$ und $x=3$ immer positiv und kann als bestinmende Function gebraucht werden.

Es ist

$$
\begin{aligned}
& {[0]=+-+} \\
& {[3]=+--;}
\end{aligned}
$$

mithin ist zwischen diesen Grenzen eine Wurzel der Gleichung $f x=0$ enthalten. Zieht man die Grenzen enger zusammen, damit sie nur um eine 
Decimal-Einheit verschieden sind, so findet man

$$
\begin{aligned}
& \left.[1]=\begin{array}{ccc}
+ & \overline{0} & + \\
0,35 & 0,57 & 0,2 \\
++ & \overline{0} & \overline{0,23}
\end{array}\right] \\
& {[2]=}
\end{aligned}
$$

Hier ist 1 die äufsere Grenze. Nun ist $\frac{0,35}{2.0,28}=0,6$; also $k=0$, und aufserdem $n=0$; mithin sind die Grenzen nicht eng genug zur nähernden Berechung. Setzt man $x=1,4$ und $x=1,5$, so findet malr

$$
\begin{aligned}
& {[1,4]=\underset{0,303}{+} \overline{0,445} \quad+\frac{+}{0,020}} \\
& {[1,5]=\underset{0,292}{+} \quad \overline{415} \quad \overline{0,432} \text {. }}
\end{aligned}
$$

Nun ist $\frac{0,303}{2.0,415}=0,3$, also $k=0, n=1$; mithin sind die Grenzen eng genug zur nähernden Berechnung. Der Quotient $\frac{0,020}{0,445}$, auf zwei Decimalstellen berechnet, giebt 0,04; also ist der erste Näherungsuerth 1,4+0,05= 1,45. Dieser Werth ist zu grofs und die Wurzel ist zwischen 1,44 und 1,45 enthalten. Da 1,44 die äufsere Grenze ist, so mufs man den Quotienten $\frac{f(1,44)}{f^{\prime}(1,44)}$ auf vier Decimalstellen entwickeln. Dieses giebt $\frac{0,002508}{0,433 t}=0,005 \%$; also ist der zweite Näherungswerth $1,44+0,0058=1,4458$ bis auf $\left(\frac{1}{10}\right)$ genau. Auch Poisson findet 1,4457.

Die nächste Wurzel findet man an leichtesten durch folgende Betrachtungen. So wie früher nachgewiesen wurde, dafs die Function $f^{\prime \prime} x$ zwischen den Grenzen $x=0$ und $x=3$ immer positiv ist, so lassen sich auch leicht ähnliche, aber weitere Grenzen angeben, zwischen welchen die folgenden Differentialquotienten beständig dasselbe Zeichen habeu. Demn man hat
4. $f^{\mathrm{III}} x=-\left(\frac{1}{2.3}-\frac{x}{2.3 .4}+\frac{x^{2}}{2^{2} .3 .4 .5}-\frac{x^{3}}{2^{2} \cdot 3^{2} .4 .5 .6}+\frac{x^{4}}{2^{2} .3^{2} .4^{2} .5 .6 .7}-\ldots ..\right)$,
5. $\left.f^{\mathrm{IV}} x=\frac{1}{2.3 .4}-\frac{x}{2.3 .4 .5}+\frac{x^{2}}{2^{2} .3 .4 .5 .6}-\frac{x^{3}}{2^{2} .3^{2} .4 .5 .67}+\ldots\right)$,
6. $f^{v} x=-\left(\frac{1}{2.3 .4 .5}-\frac{x}{2.3 .4 .5 .6}+\frac{x^{2}}{2^{2} .3 .4 .5 .6 .7}-\frac{x^{3}}{2^{2} .3^{2} \cdot 4.5 .6 .7 .8}+\ldots.\right)$,

*) Man bemerke, dafs es nur nöthig ist, die Werthe von zwei der Functionen $f^{\prime \prime} x, f^{\prime} x, f x$ zu berechnen, indem man daraus den Werth der dritten finden kann, da diese drei Funclionen durch die Gleichung

mir einander verbunden sind.

$$
f x+f^{\prime} x=-x f^{\prime \prime} x
$$


7. $f^{r \prime} x=\frac{1}{2.3 .4 .5 .6}-\frac{x}{1.3 .4 .5 .6 .7}+\frac{x^{2}}{2^{2} .3 .4 .5 .6 .7 .8}-\frac{x^{2}}{2^{2} .3^{2} .4 .5 .6 .7 .8 .9}+\ldots .$,

8. $f^{\mathrm{rI}}=-\left(\frac{1}{2.3 .4 .5 .6 .7}-\frac{x}{2.3 .45 .6 .7 .8}+\frac{x^{2}}{2^{2} .3 .4 .5 .6 .7 .8 .9}-\frac{x^{3}}{2^{2} \cdot 3^{2} 4.5 .6 .7 .8 .9 .10}+\ldots\right)$.

In der Reihe (4.) ist nun der Zahlenwerth jedes Gliedes gröfser als der des folgenden, sobald $x$ nicht gröfser als 4 ist: daher ist $f^{\text {Ix }} x$ zwischen den Grenzen $x=0$ und $x=4$ immer negativ. Eben so findet man aus den Reihen (5., 6., 7., 8.), dals $f^{\mathrm{v}} x$ zwischen den Grenzen $x=0$ und $x=5$ immer positiv ist, dafs $f^{v} x$ zwischen den Grenzen $x=0$ und $x=6$ immer negativ ist, dafs $f^{\prime \prime} x$ zwischen den Grenzen $x=0$ und $x=7$ immer positiv ist und $f^{n 1} x$ zwischen den Grenzen $x=0$ und $x=8$ immer negativ. Man kann daher jede dieser Functionen zwischen den entsprechenden

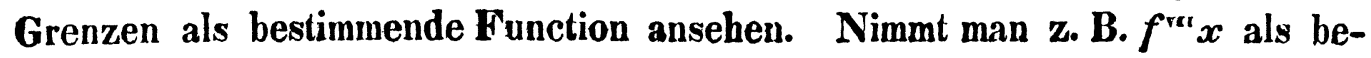
stimmende Function, so hat man

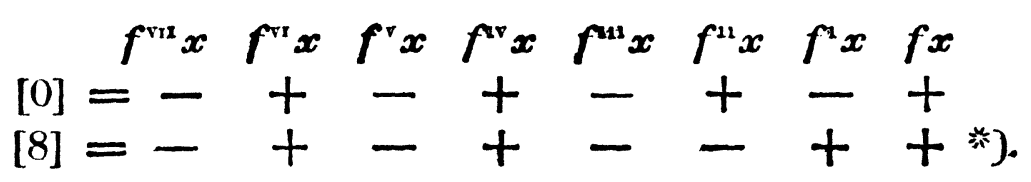

Die obere Reihe enthält 7, die untere 5 Zeichenwechsel : es sind also zwischen den Grenzen $x=0$ und $x=8$ zwei Wurzeln der Gleichung $f x=0$ enthalten. Da wir aber bereits wissen, dafs eine Wurzel, und nur eine zwischen den Grenzen $x=0$ und $x=3$ enthalten ist, so muls die andere nothwendig zwischen den Grenzen $x=3$ und $x=8$ liegen. Substituirt man allmälig die dazwischen fallenden Werthe $4,5,6,7$, so findet man, dals die Wurzel zwischen 7 und 8 enthalten ist; denn es ist

$$
[\tau]=-+-+-+-
$$

das heifst die Zeichenreihe [7] hat 6 Zeichenwechsel, während die Zeichenreilhe [8] nur 5 hat. Zugleich ergiebt sich aus dem Vergleiche der zwei Reihen [7] und [8], dars die Gleichung $f^{t x} x=0$ zwischen den Grenzen $x=7$ und $x=8$ keine Wurzel hat und dafs also $f^{\prime \prime} x$ zwischen diesen Grenzen als bestimmende Function gebraucht werden kann. Und zwar

*) Da je drei aufeinanderfolgende Differentialquotienten $f^{n} x, f^{n+1} x, f^{n+2} x$ durch die Gleichung

$$
f^{n} x+(n+1) f^{n+1} x+x f^{n+2} x=0
$$

mit einander verbunden sind, so ist klar, dafs man die Werthe aller folgenden Differentialquotienten unmittelbar aus den Werthen von $f x$ und $f^{\prime} x$ finden kann. 
hat man

$$
\begin{aligned}
& {[\gamma]=\overline{0,007} \quad+\quad+\frac{13}{0,07}} \\
& {[8]=\frac{-}{0,02} \underset{0,27}{+}+}
\end{aligned}
$$

Non ist $\frac{0.02}{2 \cdot 0,13}=0,0 \%$, also $k=1$; auch ist $n=0$; mithin die Bedingung $n=1-k$ erfüllt. Hier ist 7 die äufsere Grenze und es mufs der Quotient $\frac{0,07}{0,13}$ auf eine Decimalstelle entwickelt werden. Dies giebt 0,5 ; also ist der erste Näherungswerth $7+0,6=7,6$. Dieser Werth ist zu klein; mithin liegt die Wurzel zwischen 7,6 und 7,7. Ferner hat man, wenn man den Quotienten $\frac{f(7,6)}{f^{\prime}(7,6)}=\frac{0,002}{0,123}$ anf drei Decimalstellen entwickelt, 0,016; also ist der sweite Näherungswerth 7,6 + 0,017 = 7,61\%. Dieser Werth ist zu klein. Die Wurzel liegt mithin zwischen 7,617 und 7,618. Der Quotient $\frac{f(7,617)}{f^{\prime}(7,617)}$ $=\frac{0,0001005}{0,12326}$ kann auf 7 Stellen entwickelt werden. Dieses giebt 0,0008153; also ist der dritte Näherungsucerth 7,6178153. Poisson findet 7,6243, welcher Werth schon in der zweiten Decimalstelle unrichtig ist.

Auf dieselbe Weise können nun auch die übrigen Wurzeln leicht mit Hülfe der höheren Differentialquotienten gefunden werden.

28. Die Gleichung

$$
F \cdot x=1-\frac{x}{2}+\frac{x^{2}}{3.2^{2}}-\frac{x^{3}}{4(2.3)^{2}}+\frac{x^{4}}{5(2.3 .4)^{2}} \ldots=0
$$

welche in der Theorie der Bewegung eines elastischen Membrans vorkomnt, steht mit der vorhergehenden in genauer Verbindung, indem $\boldsymbol{F} x=-f^{\prime} x$ ist. Diese Gleichung hat ebenfalls nur positive Wurzeln und es ergiebt sich aus den im Vorbergehenden angestellten Betrachtungen, dafs $F^{\prime \prime} x$ zwischen den Grenzen $x=0$ und $x=4$ immer positiv ist und mithin zwischen diesen Grenzen als bestimmende Function gebraucht werden kann. Man findet

$$
\begin{aligned}
& {[0]=+-+} \\
& {[4]=++-}
\end{aligned}
$$

Offenbar liegt also eine Wurzel der Gleichung zwischen den Grenzen $x=0$ und $x=4$. Da aber auch eine Wurzel der Gleichung $F^{\prime} x=0$ zwischen diesen Grenzen liegt, so muis man engere Grenzen nehmen. Man findet

$$
\begin{aligned}
& {[3]=t-t} \\
& {[4]=t+-}
\end{aligned}
$$


Diese Grenzen sind noch nicht eng genug. Dagegen hat man

$$
\begin{aligned}
& {[3,6]=\underset{0,06}{+} \underset{0,11}{+} \stackrel{+}{0,007}} \\
& {[3, \bar{\gamma}]=\underset{0,05}{+} \overline{0,10} \quad \overline{0,003} \text {. }}
\end{aligned}
$$

Hier ist $\frac{0,06}{2 \cdot 0,10}=0,3$, also $k=0, n=1$. Die äufsere Grenze ist 3,6 und man hat $\frac{0,007}{0,11}=0,06$; also ist der erste Nüherungswerth $3,6+0,07=$ 3,67. Dieser Werth ist zu klein; also ist die Wurzel zwischen 3,67 und 3,68 enthalten. Ferner ist $\frac{F(3,67)}{F^{\prime}(3,67)}=\frac{0,000053}{0,1097}=0,0004$, also der zweite Nüherungswerth 3,6\%05 bis auf $\left({ }_{1}^{\frac{3}{0}}\right)^{4}$ genau. Statt dieses Werthes hat Poisson den Werth 3,55, der schon in der ersten Decimalstelle abweicht *).

Um die nächste Wurzel zu finden, betrachte man die höheren Differentialquotienten. Es ergiebt sich aus dem Früheren, dafs allgemein $\boldsymbol{F}^{2 m} \boldsymbol{x}$ positiv ist zwischen den Grenzen $x=0$ und $x=2 m+2$, und dafs $F^{2 m+1} x$ negativ ist zwischen den Grenzen $x=0, x=2 m+3$. Diese Functionen kömnen also zwischen den entsprechenden Grenzen als bestimmende gebraucht werden. Nimmt man z. B. die Function $F^{x 1} x$ zur bestimmenden, so weifs man, dafs diese Function zwischen den Grenzen $x=0$ und $x=13$ beständig negativ ist. Nun ist

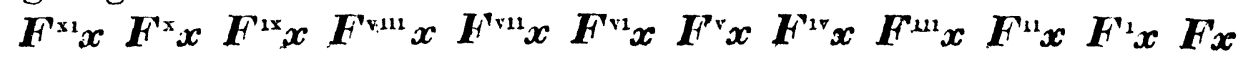

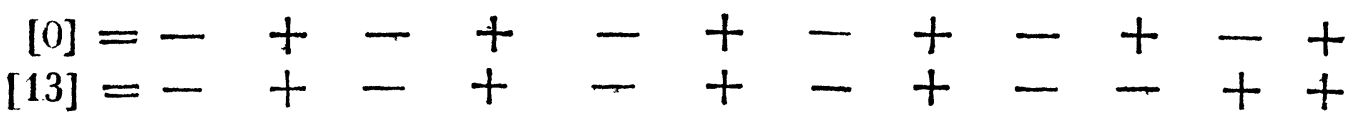

Die Zeichenreihe [0] enthält 11 Zeichenwechsel, die Zeichenreihe [13] nur 9. Es werden also zwischen den Grenzen 0 und 13 zwei Wurzeln der Gleichung $\boldsymbol{F} x=0$ angedeutet, und da man schon weifs, dafs eine dieser Wurzeln zwischen 0 und 4 enthalten ist, so mufs die andere zwischen 4 und 13 liegen.

Uebrigens wäre es nicht ıöthig gewesen, bis zur Function $F_{x}$ zurückzugehen, um zu finden, wie viele Wurzeln zwischen den Grenzen 0 und 13 liegen. Jas vorstehende Schema zeigt nämlich, dafs die Gleichung $F^{\mathrm{II}} x=0$ zwischen den Grenzen 0 und 13 keine Wurzel hat, und dafs $\boldsymbol{F}^{\text {us }} x$ zwischen diesen Greuzen bestäudig negativ ist. Dies hätte

*) In der angefürten Abhandlung p. 506. 
man auch unmittelbar aus der Reihe finden können, welche den Werth von $\boldsymbol{F}^{\text {"I }} \boldsymbol{x}$ angiebt. Substituirt man in derselben statt $x$ den Werth 13, so ist zwar dás erste Glied $\frac{1}{2.3 .4}$ kleiner als das darauf folgende $\frac{13}{2.3 .4 .5}$ : bei den spätern Gliedern ist aber jedes positive Glied gröfser als das darauf folgeude negative; mithin ist die Summe dieser Glieder positiv. Nun ist aber schon der Werth der Summe der ersten Glieder

$\frac{1}{2.3 .4}-\frac{13}{2.3 .4 .5}+\frac{13^{2}}{2^{2} \cdot 3.4 .5 .6}-\frac{13^{3}}{2^{2} \cdot 3^{2} \cdot 4.5 .6 .7}+\frac{13^{4}}{2^{2} .3^{2} \cdot 4^{2} \cdot 5.6 .7 .8}-\frac{13^{5}}{2^{2} \cdot 3^{2} .4^{2} \cdot 5^{2} \cdot 6.7 .8 .9}$ positiv: mithin ist um so mehr $-F^{m} 13$ positiv oder $F^{m} 13$ negativ; und eben so ist es bei den kleineren Zahlen. Um die zweite Wurzel zu finden, braucht man daher nur das Schema

$$
\begin{aligned}
& {[4]=-\boldsymbol{F}^{\prime \prime} \boldsymbol{F}^{\prime \prime} \boldsymbol{x} \boldsymbol{F}^{\mathrm{L}} \boldsymbol{x} \boldsymbol{F}} \\
& {[13]=-+++}
\end{aligned}
$$

zu betrachten. Da aber $\boldsymbol{F}^{11} x$ zwischen diesen Grenzen eine Wurzel hat, so mufs man die Grenzen enger ziehen. Man findet

$$
\begin{aligned}
& \text { [12] }=\overrightarrow{0,003}_{0,025}^{+} \overrightarrow{0,007} \\
& {[13]=\overline{0,004}_{0,021}^{+}+}
\end{aligned}
$$

Hier ist $\frac{0,00 t}{2.0,02 t}=0,9$; also ist $k=0, n=0$. Die Grenzen sind daher nicht eng genug zur nähernden Berechnung.

Man findet ferner

$$
\begin{aligned}
& {[12,3]=\overline{0,004}_{0,024}^{+} \underset{0,000113}{+}} \\
& {[12,4]=\overline{0,004}_{0,024}^{+}+}
\end{aligned}
$$

Es ist $\frac{0,004}{2.0,024}=0,08$, also $k=1$; auch ist $n=1$, mithin die Bedingung $n>1-k$ erfüllt. Da $2 n+k=3$ ist, so mufs der Quotient $\frac{0,000113}{0,024}$ auf drei Decimalstellen entwickelt werden. Dies giebt 0,004; also ist der erste Nüherungswerth 12,305. Dieser Werth ist zu grofs; mithin ist die Wurzel zwischen 12,304 und 12,305 enthalten. Der nächste Näherungswerth kann uun auf Decimalstellen genau gefunden werden. Es ist

$$
\frac{F(12,304)}{F^{\prime}(12,304)}=\frac{0,000014980}{0,024393}=0,0006141 ;
$$

also ist der nächste Näherungswerth 12,3046142. Poisson findet den Werth 12,41 (ebend.).

Crelle's Journal d. M. Bd. XXII. Hft. 1. 
29. Diese Beispiele werden genügen, um zu zeigen, wie man in ähnlichen Fälleu zu verfahren hat, und un zu beweisen, dafs man vermittelst der angegebenen Methode alle reellen Wurzeln der transcendenten Gleichungen mit jedem beliebigen Grade von Genauigkeit finden kann. Ich will nun schliefslich noch eine besonders interessante Frage berühren, nemlich die, wie man erkennen kann, ob eine transcendente Gleichung nur reelle Wurzeln hat. Auf diese Frage ist man besonders durch mathematischphysikalische Untersuchungen gekommen, welche bänfig anf transcendente Gleichungen führen, und wo es sich oft unmittelbar aus der Natur der Untersuchung selbst ergiebt, dafs solche Gleichungen keine imaginären Wurzeln haben. Es kommt darauf an, dasselbe auf rein analytischem Wege nachzuweisen. Allgemeine Regeln, vermitlelst deren man in jedem Falle unmittelbar aus dem Bau der Gleichung entscheiden köunte, ob eine transcendente Gleichung nur reelle Wurzeln hat, giebt es bis jetzt nicht; und dies darf um so weniger auffallen, da die ähnliche Untersuchung in Beziehung auf die algebraischen Gleichungen ebenfalls nicht ohne Schwierigkeiten ist. Durch verschiedene besondere Betrachtungen kann man aber allerdings von einer Menge transcendenter Gleichungen beweisen, dafs sie nur reelle Wurzeln haben. Am einfachsten geschieht es natürlich in dem Falle, wenn eine Gleichung $f x=0$ gegeben ist und man weils, dafs $f x$ das Product einer Anzahl einfacher Factoren ist. die alle reellen Wurzeln der gegebenen Gleichung entsprechen. So z. B. hat die Gleichung $\sin x=0$ nothwendig nur reelle Wurzeln, da

$$
\sin x=y\left(1-\frac{x^{2}}{\eta^{2}}\right)\left(1-\frac{x^{2}}{4 \pi^{2}}\right)\left(1-\frac{x^{2}}{9 \pi^{2}}\right) \ldots
$$

ist; dasselbe ist bei der Gleichung $\cos x=0$ der Fall.

Eine andere selhr fruchtbare Betrachtungsweise besteht darin, dafs man in der gegebenen Gleichung $f x=0$ statt $x$ seines Werths $a+b \sqrt{ }-1$ substituirt, wo $a$ und $b$ reelle Gröfsen bedeuten, und dann nachweiset, dafs die Annahme auf Widersprüche führt, wenn man nicht $b=0$ setzt. Diese Beweisfïhrung scheint zuerst Fourier angewendet zu haben, welcher bemerkt ${ }^{*}$ ), dals es hinreiche, den Werth $a+b \sqrt{ }-1$ statt $x$ in die Gleichung $x-\lambda \operatorname{tang} x=0$ zu substituiren, wo $\lambda$ eine Zahl bedeutet, die kleiner als die Einheit ist, um zu beweisen, dafs die Gleichung keine Wurzel dieser Art haben könne. Später bat Cauchy in einer besondereu Abhandlung

*) Théorie de la chaleur p. 366 . 
diese Idee ausführlich behandelt und auf eine grofse Anzahl von Gleichungen angewendet*). Indem ich auf diese Abhandlung verweise, werde ich mich begnügen, hier noch einige Gleichungen abzuhandeln, die nicht bei Cauchy vorkommen, und Gelegenheit finden, dabei noch einige besondere Kunstgriffe anzuwenden. Ich werde besonders die im Früheren abgehandelten Gleichungen berücksichtigeu.

Die Gleichung

$$
\sin x=a
$$

kann keine imaginären Wurzeln von der Form $y+z \checkmark-1$ haben, wenn $a$ eine Zahl bedeutet, die nicht gröfser als die Einheit ist. Denn substituirt man statt $x$ den Werth $y+z \sqrt{ }-1$, so erhält man die zwei Gleichungen

$$
\begin{aligned}
& \text { 1. } \quad \sin y \cdot \frac{1}{2}\left(e^{z}+e^{-z}\right)=a, \\
& \text { 2. } \cos y \cdot \frac{1}{2}\left(e^{z}-e^{-z}\right)=0 .
\end{aligned}
$$

Soll nun $z$ nicht 0 sein, so kann man der zweiten Gleichung nur dann Genüge leisten, wenn man $\cos y=0$ setzt. In diesem Falle ist aber siny $=1$, also ginge die Gleichung (1.) in folgende über

$$
\text { 3. } \frac{1}{2}\left(e^{z}+e^{-z}\right)=a
$$

Nun ist aber

$$
\frac{1}{2}\left(e^{z}+e^{-z}\right)=1+\frac{1}{2} z^{2}+\frac{z^{4}}{2.3 .4}+\ldots>1:
$$

also kann die Gleichung (3.) nicht bestehen, wenn $a$ der Einheit gleich oder kleiner als dieselbe ist. Der Beweis gilt auch noch in dem Falle, weun $y=0$ ist.

Auch die Gleichung

$$
\cos x=a
$$

kann keine imaginären Wurzeln von der Form $x=y+z r-1$ haben, wenn $a$ nicht gröfser als die Einheit ist. Denn substituirt man diesen Werth statt $x$, so findet man die zwei Gleichungen

4. $\quad \begin{aligned} \cos y \cdot \frac{1}{2}\left(e^{z}+e^{-z}\right) & =a, \\ \text { 5. } \quad \sin y \cdot \frac{1}{2}\left(e^{z}-e^{-z}\right) & =0 \text {. }\end{aligned}$

Soll nun $z$ nicht gleich Null sein, so mufs $\sin y=0$, also $\cos y=1$ sein; mithin hat man wieder

$$
\frac{1}{2}\left(e^{\tau}+e^{-x}\right)=a ;
$$

welche Gleichung nicht bestehen kann, wenn $a \overline{\overline{<}} 1$ ist.

Auch die in \$. 24. abgehandelte Gleichung

$$
\left(4-3 x^{2}\right) \sin x-4 x \cos x=0
$$

*) Cauchy Exercices des Mathemat. T. I. p. 297 ff. 
hat keine imaginären Wurzeln. Diese Gleichung kann auch wie folgt geschrieben werden:

$$
\text { 6. } \quad \text { tang } x=\frac{-\frac{4}{3} x}{x^{2}-\frac{4}{3}}
$$

sie ist mithin in der allgemeineren Gleichung

$$
\operatorname{tang} x=\frac{a x}{x^{2}+b}
$$

enthalten, welche bereits Cauchy in der erwähnten Abhandlung (\$. 4.) abgehandelt hat. Gerade den Fall aber, der hier zur Betrachtung kommt, wo nemlich $a$ negativ und $b=a$ ist, hat er nicht erörtert. Man kann nun zuerst beweisen, dafs die Gleichung (6.) keine imaginäre Wurzel von der Form $x=y \sqrt{ }-1$ haben kann; oder, man kann noch allgemeiner beweisen, dals die Gleichung

$$
\text { 7. tang } x=-\frac{a x}{x^{2}-a}
$$

keine solche Wurzel haben kann, sobald $a \$ 3$ ist. Denn setzt man in (7.) statt $x$ den Werth $y \checkmark-1$, so erbält man

$$
\frac{a}{a+y^{2}}=\frac{1+\frac{y^{2}}{1.2 .3}+\frac{y^{4}}{1.2 .3 .4 .5}+\ldots}{1+\frac{y^{2}}{1.2}+\frac{y^{4}}{1.2 .3 .4}+\ldots}
$$

oder

8. $\left.\left.\quad a+\frac{a y^{2}}{1.2}+\frac{a y^{4}}{1.2 .3 .4}+\ldots=a+\frac{a}{1.2 .3}+1\right\} \begin{array}{c}y^{2}+\frac{a}{1.2 .3 .4 .5} \\ +\frac{1}{1.2 .3}\end{array}\right\} y^{4}+\ldots$

Ist nun aber $a<3$, so ist offenbar der Coefficient jeder Potenz von $y$ in der ersten Reihe kleiner als der Coefficient der entsprechenden Potenz in der zweiten, nemlich

$$
\begin{aligned}
\frac{a}{1.2} & <1+\frac{a}{1.2 .3}, \\
\frac{a}{1.2 .3 .4} & <\frac{1}{1.2 .3}+\frac{a}{1.2 .3 .4 .5}, \\
\frac{a}{1.2 .3 .4 .5 .6} & <\frac{1}{1.2 .3 .4 .5}+\frac{a}{1.2 .3 .4 .5 .6 .7} \\
\text { etc. } &
\end{aligned}
$$

und die Gleichung (8.) kann daher unter diesen Umständen nicht Statt finden. Auf dieselbe Weise läfst sich auch zeigen, dafs die Gleichung

$$
\operatorname{tang} x=\frac{-a x}{x^{2}-b}
$$

keine imaginäre Wurzel von der Form $y \checkmark-1$ hat, sobald $a<3$ and $b>a$ ist. 
1. Stern, über die Auflösung der transcendenten Gleichungen.

Die Gleichung (7.) kann aber auch keine Wurzel von der Form $x=y+z \sqrt{ }-1$ haben, sobald $a$ kleiner als 3 ist. Um dies zu beweisen, setze man $y+z r-1=\rho(\cos \alpha+\sin \alpha r-1)$ und substituire diesen Werth statt $x$. Dieses giebt

$$
\begin{gathered}
\frac{\operatorname{tg} x}{x}=\frac{1-\frac{\rho^{2}(\cos 2 \alpha+\sin 2 \alpha \sqrt{ }-1)}{1.2 .3}+\frac{\varrho^{4}(\cos 4 \alpha+\sin 4 \alpha \sqrt{ }-1)}{1.2 .3 .4 .5}-\frac{\rho^{\theta}(\cos 6 \alpha+\sin 6 \alpha \sqrt{ }-1)}{1.2 .3 \ldots 7} \ldots .}{1-\frac{\varrho^{2}(\cos 2 \alpha+\sin 2 \alpha \sqrt{-1})}{1.2}+\frac{\rho^{4}(\cos 4 \alpha+\sin 4 \alpha \sqrt{-1})}{1.2 .3 .4}-\frac{\rho^{6}(\cos 6 \alpha+\sin 6 \alpha \sqrt{-1})}{1.2 \ldots 7} \ldots} \\
=\frac{-a}{\rho^{2}(\cos 2 \alpha}+\sin 2 \alpha \sqrt{-1)-\alpha}
\end{gathered}
$$

Hieraus findet man

$-a\left[1-\frac{\rho^{2} \cos 2 \alpha+\sin 2 \alpha \sqrt{ }-1}{1.2}+\frac{\varrho^{4}(\cos 4 \alpha+\sin 4 \alpha \sqrt{ }-1)}{1.2 .3 .4}-\frac{\varrho^{6}(\cos 6 \alpha+\sin 6 \alpha \sqrt{ }-1)}{1.2 \ldots . . .6]}\right.$

$=\rho^{2}(\cos 2 \alpha+\sin 2 \alpha \gamma-1)-\frac{\rho^{*}(\cos 4 \alpha+\sin 4 \alpha \sqrt{-1})}{1.2 .3}+\frac{\rho^{\theta}(\cos 6 \alpha+\sin 6 \alpha \sqrt{-1})}{1.2 .3 .4 .5}-\ldots$

$-a\left[1-\frac{\rho^{2}(\cos 2 \alpha+\sin 2 \alpha \sqrt{ }-1)}{1.2 .3}+\frac{\rho^{4}(\cos 4 \alpha+\sin 4 \alpha \sqrt{ }-1)}{1.2 .3 .4 .5}-\frac{\rho^{8}(\cos 6 \alpha+\sin 6 \alpha \sqrt{ }-1)}{1.2 \ldots . . .7} \ldots\right]$

und wenn man die reellen Glieder einander gleich setzt,

$$
\begin{aligned}
& \frac{a}{1.2} \rho^{2} \cos 2 \alpha-\frac{a}{1.2 .3 .4} \rho^{4} \cos 4 \alpha+\frac{a}{1.2 \ldots .6} \rho^{6} \cos 6 \alpha-\ldots \\
&=\left(1+\frac{a}{1.2 .3}\right) \rho^{2} \cos 2 \alpha-\left(\frac{1}{1.2 .3}+\frac{a}{1.2 .3 .4 .5}\right) \rho^{4} \cos 4 \alpha \\
&+\left(\frac{1}{1.2 .3 .4 .5}+\frac{a}{1.2 .3 \ldots .7}\right) \rho^{6} \cos 6 \alpha-\ldots
\end{aligned}
$$

also

$$
\begin{aligned}
\frac{a}{1.2} & =1+\frac{a}{1.2 .3}, \\
\frac{a}{2.3 .4} & =\frac{1}{1.2 .3}+\frac{a}{1.2 .3 .4 .5} \\
\text { etc. } &
\end{aligned}
$$

welche Gleichungen, wie schon früher bemerkt wurde, nicht Statt haben können, sobald $a<3$ ist.

Aus denselben Gründen folgt, dafs auch die Gleichung

$$
\text { tang } x=\frac{-a x}{x^{2}-b}
$$

keine Wurzel von der Form $y+\approx r-1$ haben kann, sobald $b>a$ und $a<3$ ist.

Die in 55. 25. und 26. abgehandelten Gleichungen

$$
\begin{aligned}
& \left(e^{x}+e^{-x}\right) \cos x-2=0, \\
& \left(e^{x}+e^{-x}\right) \cos x+2=0
\end{aligned}
$$

baben zwar, wie schon dort bemerkt wurde, imaginäre Wurzeln, deren 
reeller Theil Null ist: sie können aber keine imaginären'Wurzeln haben, von welchen der reelle Theil nicht Null ist. Ich will zuerst die Gleichung

$$
\text { 9. }\left(e^{x}+e^{-x}\right) \cos x-2=0
$$

betrachten. Man substituire statt $x$ den Werth $y+z \sqrt{-1}$, so findet mau 10. $e^{y+z \sqrt[V]{ }-1}+e^{-(y+z V-1)}=\left(e^{y}+e^{-y}\right) \cos z+\left(e^{y}-e^{-y}\right) \sin z r-1$,

11. $\cos (y+z r-1)=\frac{1}{2}\left(e^{z}+e^{-z}\right) \cos y-\frac{1}{2}\left(e^{z}-e^{-z}\right) \sin y r-1$.

Man erhält also statt der Gleichung (9.) die zwei Gleichungen

12. $\left(e^{y}+e^{-y}\right) \frac{1}{2}\left(e^{z}+e^{-z}\right) \cos y \cos z+\left(e^{y}-e^{-y}\right) \frac{1}{2}\left(e^{z}-e^{-z}\right) \sin y \sin z=2$.

13. $\left(e^{y}-e^{-y}\right) \frac{1}{2}\left(e^{z}+e^{-z}\right) \cos y \sin z-\left(e^{y}+e^{-y}\right) \frac{1}{2}\left(e^{z}-e^{-z}\right) \sin y \cos z=0$. Stalt der Gleichung (13.) kann man auch schreiben

$$
\text { 14. } \frac{e^{y}-e^{-y}}{e^{y}+e^{-y}} \cdot \frac{\cos y}{\sin y}=\frac{e^{z}-e^{-z}}{e^{z}+e^{-z}} \cdot \frac{\cos z}{\sin z} \text {. }
$$

Soll nun diese Gleichung bestehen, ohne dafs $y$ oder $z$ Null sind, so murs nothwendig $y=z$ sein. Alsdann geht aber die Gleichung (12.) in folgende über:

$$
\text { 15. }\left(e^{y}+e^{-y}\right)^{2}(\cos y)^{2}+\left(e^{y}-e^{-y}\right)^{2}(\sin y)^{2}=4 \text {, }
$$

oder, wenn man statt $(\sin y)^{2}$ seinen Werth $1-(\cos y)^{2}$ setzt, und reducirt, in

16. $\left(e^{y}-e^{-y}\right)^{2}+4(\cos y)^{2}=4$,

$$
\begin{aligned}
\text { also in }\left(e^{y}-e^{-y}\right)^{2} & =4(\sin y)^{2}, \\
\text { oder } e^{y}-e^{-y} & =2 \sin y ;
\end{aligned}
$$

welches ungereimt ist, da $e^{y}-e^{-y}$ immer gröfser als $2 \sin y$ ist.

Man sieht zugleich, dafs die Gleichung (9.) nur ein specieller Fall einer allgemeineren Gleichung ist, indem man auf dieselbe Weise zeigen kann, dars die Gleichung

$$
\left(e^{x}+e^{-x}\right) \cos x-a=0
$$

keine imaginäre Wurzel von der Form $y+z \sqrt{-1}$ bat, sobald $a$ nicht grölser als 2 ist *).

*) Die specielle Gleichung

$$
\left(e^{x}+e^{-x}\right) \cos x-2=0
$$

kann auch auf eine andere Gleichung zurückgeführt werden, von welcher schon Cauchy bewiesen hat, dafs sie keine imaginäre Wurzeln hat, von der Form $x=y+z \sqrt{ }-1$. Man hat nendich, da allgemein

$$
\operatorname{tang} \frac{1}{2} x= \pm\left(\frac{1-\cos x}{1+\cos x}\right)^{\frac{1}{3}}
$$

ist, wenn man statt $\cos x$ den Werth $\frac{2}{e^{x}+e^{-x}}$ substituirt,

$$
\text { tang } \frac{1}{2} x= \pm \frac{e^{\frac{1}{2} x}-e^{-\frac{1}{2} x}}{e^{\frac{1}{2} x}+e^{-\frac{1}{2} x}}
$$

für welche Gleichung Cauchy bewiesen hat, dals $x$ nicht $=y+z \sqrt{-1}$ sein kann. 
Aus einem noch allgemeineren Gesichtspuncte kann man die Gleichung

$$
\left(e^{x}+e^{-x}\right) \cos x+2=0
$$

betrachten, indem man die Behauptung aufstellt, dafs die Gleichung

$$
\text { 17. }\left(e^{x}+e^{-x}\right) \cos x+a=0
$$

keine imaginäre Wurzel von der Form $y+z \sqrt{-1}$ haben kann, sobald $a$ irgend eine positive Zahl bedeutet. Denn substituirt man wirklich $y+z \sqrt{-1}$ statt $x$, so erhält man die zwei Gleichungen

18. $\left(e^{y}+e^{-y}\right) \frac{1}{2}\left(e^{z}+e^{-z}\right) \cos y \cos z+\left(e^{y}-e^{-y}\right) \frac{1}{2}\left(e^{z}-e^{-z}\right) \sin y \sin z=-a$,

$$
\text { 19. } \frac{e^{y}-e^{-y}}{e^{y}+e^{-y}} \cdot \frac{\cos y}{\sin y}=\frac{e^{z}-e^{-z}}{e^{z}+e^{-z}} \cdot \frac{\cos z}{\sin z} \text {. }
$$

Aus (19.) folgt aber $y=z$; also geht die Gleichung (18.) in folgende über:

$$
\left(e^{y}+e^{-y}\right)^{2}(\cos y)^{2}+\left(e^{y}-e^{-y}\right)^{2}(\sin y)^{2}=-2 a ;
$$

was ungereimt ist, da der erste Theil der Gleichung positiv und der zweite negativ ist.

30. Die vorhergehende Beweisführung scheint, so weit sie auch ansreicht, dennoch auf Gleichungen, wie die in \$. 27. behandelte, nicht wohl anwendbar zu sein. Fourier hat aber bewiesen, dafs auch diese Gleichung keine imaginären Wurzeln hat. Die Methode, die er hierzu anwendet, ist insofern allgemeiner als die erste, dafs man nicht, wie dort, nöthig hat anzunehmen, dals die imaginären Warzeln in der Form $y+z \sqrt{-1}$ enthalten sind, sondern den Beweis gauz unabhăngig von der Form der Wurzeln führen kann; und sie erfordert um so mehr eine ausführliche Darstellung, da Fourier dieselbe nicht völlig entwickelt und man auch ihre Richtigkeit in $\mathbf{Z w e i f e l}$ gezogen hat. Für die algebraischen Gleichungen hat bekanntlich schon vorlängst de Gua ein Kennzeichen angegeben, durch welches man mit Bestimmtheit erkennen kann, dafs alle Wurzeln gewisser Gleichungen reell siud, und der darauf bezügliche Satz zeigt sich als eine sehr einfache Folgerung aus Fourier's Theorie der Gleichungen. Bei einer algebraischen Gleichung vom mten Grade, $f x=0$, enthält nemlich die Zeichenreihe $[-\infty] m$ Zeichenwechsel und die Zeichenreihe [ $\infty$ ] gar keinen Zeichenwechsel, und diese Zeichenwechsel gehen zwischen den Grenzen $-\infty$ und $+\infty$ aus zwei Gründen verloren. Es entspricht nemlich erstens jeder reellen, zwischen diesen Grenzen liegenden Wurzel ein Zeichenverlust; es können aber zweitens anch Zeichenwechsel verloren gehen, ohne dafs 
solches auf eine reelle Wurzel deutete. So viele Zeichenwechsel nun auf diese zweite Art verloren gehen: eben so viel reelle Wurzeln fehlen der Gleichung, und sie mufs daher nothwendig eben so viele imaginäre Wurzeln haben, weil jede algebraische Gleichung vom mten Grade nicht melır und nicht weniger als $m$ reelle oder imaginäre Wurzeln hat. Der Verlust von Zeichenwechseln, der nicht auf reelle Wurzeln deutet, kann aber nur in dem Falle rorkommen, wenn es einen reellen Werth $\alpha$ giebt, der so beschaffen ist, dafs zwar ${ }^{\prime}(\alpha)$ nicht Null ist, dafs aber eine der algebraischen Functionen, etwa $f^{n} x$, durch die Substitution dieses Werthes statt $x$ auf Null reducirt wird, während zu gleicher Zeit die abgeleiteten Functionen $f^{n+1} x$ und $f^{n-1} x$ dasselbe Zeichen behalten. Sobald also jeder reelle Werth von $x$, der eine der abgeleiteten Functionen auf Null reducirt, zwei Ausdrücke mit entgegengesetztem Zeichen giebt, wenn man ihn in die vorhergehende und in die folgende Function substituirt, so kann die algebraische Gleichung nur reelle Wurzeln haben. Dies ist der de Gua'sche Satz, den auch Fourier in seinem Werke ausführlich abgehandelt hat. Diese Betrachtungen lassen sich aber nicht unmittelbar auf die transcendenten Gleichungen ausdehnen. Ich habe zwar im Frülseren gezeigt, dafs die Grenzen der reellen Wurzeln der transcendenten Gleichungen auf ganz ähnliche Weise gefunden werden, wie die der algebraischen Gleichungen: ich habe aber zugleich auf einen Unterschied zwischen den algebraischen und den transcendenten Gleichungen aufmerksam gemacht, welcher im Wesentlichen darin besteht, dafs der erste Theil einer algebraischen Gleichung immer eine continuirliche Grölse und zugleich das Product einer Anzahl einfacher reeller oder imaginärer Factoren ist, die den Wurzeln dieser Gleichung entsprechen, während der erste Theil einer transcendenten Gleichung auch eine discontinuirliche Gröfse sein und vou dem Producte der einfachen Factoren, die den Wurzeln entsprechen, wesentlich verschieden sein kann. Hieraus folgt schon, dafs man bei diesen Gleichungen von der Abwesenheit reeller Wurzeln nicht auf das Vorhandensein imaginärer, und ungekehrt, schliefsen darf. Es wurde ferner früher gezeigt, dafs man die Grenzen, zwischen welchen die reellen Wurzeln liegen, auf folgende Weise findet. Man sucht zuerst die Grenzen $\alpha$ und $\beta$, zwischen welchen eine der abgeleiteten Functionen, etwa $f^{m x} x$, immer dasselbe Zeichen behält, und bildet alsdann die zwei Zeichenreihen $[\alpha]$ und $[\beta]$, so entspricht jeder reellen Wurzel, die zwischen diesen Grenzen enthalten ist, der Verlust eines Zeicheuwechsels, den die Reihe [ $\beta$ 
weniger enthält als [a]. Es können aber, wie gezeigt wurde, auch hier Zeichenwechsel verloren gehen, ohne das solches auf reelle Wurzeln deutete; jedoch nur in dem Falle, wenn ein zwischen'diesen Grenzen liegender Werth eine der in Betracht kommenden abgeleiteten Functionen auf Null reducirt, während er der vorhergehenden und der folgenden Function, wenn er in dieselben substituirt wird, gleiche Zeichen giebt. Enthält nun die Zeichenreibe [ $\beta$ ] etwa $2 n$ Zeichenwechsel weniger als [a], und weirs man, dars zwischen den Grenzen $\alpha$ und $\beta$ keine reelle Wurzel liegt, so darf man hieraus nicht, wie bei den algebraischen Gleichungen, schliefsen, dals die Gleichung $2 n$ imaginäre Wurzeln habe, weil hier die Abwesenheit der reellen Wurzeln durchaus nicht auf das Vorhandensein imaginärer deutet. Weifs man umgekehrt, dafs es keinen Werth giebt, der eine der abgeleiteten Functionen auf Null reducirt, während er, in die vorhergehende und folgende substituirt, Resultate von gleichem Zeichen giebt, so darf man daraus nicht schliefsen, dafs die transcendente Gleichung nur reelle Wurzelu habe: es kann vielmehr sein, dafs weder reelle noch imaginäre Wurzeln vorhanden sind. Anders aber verhält es sich, wenn man mit Bestimmtheit weils, dafs die transcendente Gleichung $f x=0$ so beschaffen ist, dafs $f x$ das Product einer unendlichen Anzahl einfacher, reeller oder imaginärer Factoren ist, die eben so vielen Wurzeln der Gleichung entsprechen. In diesem Falle ist $f x$, und jeder daraus abgeleitete Differentialquotient, für jeden Werth von $x$ eine continuirliche Gröfse. Sind nun z. B. $\alpha$ und $\beta$ zwei bestimmende Grenzen, und enthält die Zeichenreihe [b] eine Anzahl $\delta$ von Zeichenwechseln weniger als die Zeichenreihe $[\alpha]$, so kann die Gleichung zwischen diesen Greuzen nicht mehr als $\delta$ reelle Wurzeln haben; sind dagegen weniger als $\delta$ reelle Wurzeln zwischen diesen Grenzen enthalten, so müssen nothwendig mehrere Zeichenwechsel zwischen den Grenzen $\alpha$ und $\beta$ verloren gehen, deren Verlust nicht auf reelle Wurzeln deutet, das heifst, es müssen eine oder mehrere der abgeleiteten Functionen durch einen zwischen $\alpha$ und $\beta$ liegenden Werth auf Null reducirt werden, während die vorhergehende und die folgende Function dasselbe Zeichen behält. Sobald es also keinen solchen Werth giebt, so müssen die sämmtlichen $\delta$ Wurzeln reell sein. Dieselbe Betrachtung gilt für jede zwei andere bestimmende Grenzen. Sobald es also gar keinen reellen Werth von $x$ giebt, der so beschaffen ist, dafs er, während er eine abgeleitete Function auf Null reducirt, der vorhergehenden und den folgenden gleiche Zeichen giebt, so kann 
auch die Gleichung keine imaginäre Wurzel haben, sondern die Wurzeln müssen sämmtlich reell sein.

Auf diesem Wege hat Fourier bewiesen, dafs die Gleichung

$$
\text { 1. } f x=1-x+\frac{x^{2}}{2^{2}}-\frac{x^{3}}{2^{2} \cdot 3^{2}}+\frac{x^{4}}{2^{2} \cdot 3^{2} \cdot 4^{2}}-\ldots=0
$$

keine imaginäre Wurzeln hat. Es wurde schon früher bemerkt (\$. 27.), dafs je drei auf einander folgende Differentialquotienten durch die Gleichung

$$
\text { A. } f^{n} x+(n+1) f^{n+1} x+x f^{n+2} x=0
$$

mit einander verbunden sind. Es ist nun einleuchtend, dals weder $f x$ noch irgend eine der abgeleiteten Functionen $f^{\prime} x, f^{\prime \prime} x, \ldots$ durch einen negativen Werth von $x$ auf Null reducirt werden können. Nimmt man nun an, dafs $f^{n+1} x$ durch irgend einen reellen positiven Werth von $x$ auf Null reducirt wird, so folgt aus der Gleichung (A.)

$$
f^{n} x=-x f^{n+2} x
$$

Hieraus folgt also, dafs jeder reelle Werth von $x$, der eine der abgeleiteten Functionen auf Null reducirt, der vorhergehenden und folgenden Function verschiedene Zeichen giebt: Man kann num ferner zeigen, dafs $f x$ das Product einer unendlichen Zahl einfacher Factoren ist. Fourier thut dies, indem er die transcendente Gleichung auf eine algebraische zurück führt. Diese Gleichung heifst

$$
\text { 2. } F y=1-n y+\frac{n \cdot n-1}{1.2} \cdot \frac{y^{2}}{2}-\frac{n \cdot(n-1)(n-2)}{1.2 \cdot 3} \cdot \frac{y^{3}}{2.3}+\ldots=0 \text {, }
$$

wo $n$ eine ganze Zahl ist. Die Anzahl der Glieder dieser Gleichung ist $n+1$, und wenn man $n$ unendlich grofs setzt, so geht sie in die Gleichung (1.) über, sobald man $n y=x$ setzt. Da nun die Gleichung (2.), als eine algebraische, sich immer in Factoren zerlegen läfst, wie grofs auch $n$ sein mag, so folgt hieraus, dafs es auch noch der Fall ist, wenn $n$ unendlich grofs ist, das heifst, es mufs auch $f x$ das Product einer unendlichen Zahl von Factoren sein, die den Wurzeln der Gleichung $f x=0$ entsprechen. Diese Factoren müssen aber sämmtlich reell sein; wie aus dem Zusammenhange der Differentialquotienten folgt; und es ist daher bewiesen, dafs $f x$ das Product von lauter reellen Factoren ist, die den Wurzeln der Gleichung $f x=0$ entsprechen.

Auf dieselbe Weise könnte man auch, wenn es nicht schon bekannt wäre, zeigen, dafs die Functionen $\sin x$ und $\cos x$ aus dem Producte einfacher reeller Factoren zusammengesetzt sind, das heifst, dafs die Gleichungen $\sin x=0$ und $\cos x=0$ keine imaginären Wurzeln haben. Denn 
die Gleichung

3. $f x=\sin x=x-\frac{x^{3}}{1.2 .3}+\frac{x^{3}}{1.2 .3 .4 .5}-\ldots=0$

ist nur ein besonderer Fall der allgemeinen Gleichung

4. $F y=n y-\frac{n(n-1)(n-2)}{1.2 .3} y^{3}+\frac{n \cdot(n-1)(n-2)(n-3)(n-4)}{1.2 .3 .4 .5} y^{5}-\ldots=0$, inden man in der Gleichung (4.) nur $n=\infty$ und $n y=x$ zu setzen braucht, um die Gleichung (3.) zu erhalten. Da nun die Gleichung (4.) immer in Factoren zerlegbar ist, so folgt das Nemliche für die Gleichung (3.). Nun ist ferner

$$
\begin{aligned}
f x & =\sin x, \\
f^{2} x & =\cos x, \\
f^{11} x & =-\sin x, \\
f^{111} x & =-\cos x, \\
f^{\mathrm{t}} x & =\sin x:
\end{aligned}
$$

es ist also allgemein $f^{n} x=-f^{n+2} x$, und diese Ausdrücke haben also auch entgegengesetzte Zeichen, wenn man $f^{n+1} x=0$ setzt. Hieraus folgt, dafs sin $x=0$ nur reelle Wurzeln hat. Dasselbe folgt auch für die Gleichung $\cos x=0$.

31. Dieses von Fourier aufgestellte Princip, um die Realität sämmtlicher Wurzeln gewisser transcendenten Gleichungen zu erkennen, von welchem Cauchy sagt *), dafs es mehreren Schwierigkeiten unterworfen sei, ist von Poisson gradezu für falsch erklärt worden. Poisson will dies durch folgendes Beispiel beweisen. Es sei gegeben die Gleichung

$$
f x=e^{x}-b e^{a x}=e^{x}\left(1-b e^{(a-1) x}\right),
$$

wo $a$ und $b$ bekannte Gröfsen sind und $a$ positiv ist. Diese Gleichung hat nun offenbar unendlich viele imaginäre Wurzeln, die sämmtlich in der Form

$$
x=\frac{\log b+2 i \pi V-1}{1-a}
$$

enthalten sind. Differentiirt man aber, so findet man

$$
\begin{aligned}
f^{n} x & =e^{x}-b a^{n} e^{a x}, \\
f^{n+1} x & =e^{x}-b a^{n+1} e^{a x}, \\
f^{n+2} x & =e^{x}-b a^{n+2} e^{a x} .
\end{aligned}
$$

Setzt man nun $f^{n+1} x=0$, so ergiebt sich

$$
\begin{gathered}
f^{n} x=-b(1-a) a^{n} e^{a x} \\
f^{n+2} x=+b(1-a) a^{n+1} e^{a x}
\end{gathered}
$$

") Exercices des mathém. T. I. p. 338. 
folglich $f^{n} x \cdot f^{x+2} x=-b^{2}(1-a)^{2} \cdot a^{2 n+1} \cdot e^{2 a x}$, welcher Werth für jeden reellen Werth von $x$ negativ ist. Hieraus würde also folgen, dafs jedle reelle Wurzel von $f^{n+1} x$, welche man in die zwei Functionen $f^{n} x$ und $f^{n+2} x$ substituirt, Resultate von verschiedenem $Z$ Zeichen giebt, und es würde milhin nach Foutrier's Regel folgen, dafs die Gleichung $f x=0$ keine imaginären Wurzeln habe; was doch nicht der Fall ist. Hier ist aber wohl ein Trugschlufs. Der Ausdruck $f^{n+1} x$ enthält nemlich den Factor $e^{x}$, und wird Null, sobald dieser Factor Null wird: $e^{x}$ wird aber Null, sobald man $x=-\infty$ setzt. Die Gleichung $e^{x}=0$ hat mithin unendlich viele reelle Wurzeln, welche sämmtlich in dem Ausdruck $x=-\infty$ enthalten sind. Substituirt man aber einen dieser reellen Werthe in $f^{n} x$ und $f^{n+2} x$, so werden diese Functionen ebenfalls auf Null reducirt und man kann daher in diesem Falle nicht sagen, dafs sie verschiedene Zeichen haben. Es ist also nicht richtig, dafs jeder reelle Werth von $x$, der $f^{n+1} x$ auf Null reducirt, den Functionen $f^{n} x$ und $f^{n+1} x$ verschiedene Zeichen giebt, sondern es gilt dies nur für den reellen Werth, der sich aus dem Factor $1-b a^{n+1} e^{(n-1) x}$ ergiebt. Dagegeu giebt es unendlich viele Werthe von $x$, welche $f^{n+1} x$ auf Null reduciren und die dennoch, in $f^{n} x$ und $f^{n+2} x$ substituirt, nicht Resultate von verschiedenen Zeichen hervorbringen. Daher darf man auch nicht schliefsen, dafs die Gleichung $e^{x}-b e^{a x}=0$ nur reelle Wurzeln haben könne ${ }^{x}$.

32. Eine dritte, sehr scharfsinnige Methode hat Poisson mehrfach angewendet, um die Abwesenheit der imaginären Wurzeln in gewissen transcendenten Gleichungen nachzuweisen. Diese Methode ist aber schon deswegen in ihrer Anwendung beschränkt, weil sie die Abwesenheit der imaginären Wurzeln nicht unmittelbar aus der Natur der Gleichung beweiset, sondern nur da anwendbar ist, wo man durch besondere Untersuchungen, die zu einer solchen transcendenten Gleichung geführt haben, zugleich einen anderen Ausdruck erbält, welchen man zum Beweise gebrauchen kann. Auch findet man vermittelst dieser Methode nur, dafs die transcendente Gleichung keine imaginären $W$ urzeln hat, die aus einem reellen und einem imaginären Theile bestehen, also in der Form $a+b \sqrt{ }-1$ enthalten sind, wo weder $a$

*) Fourier hat den hier besprochenen Lehrsatz zuerst in seinem Werke Théorie de la chaleur p. 372 kurz vorgetragen. Poisson hat darauf in dem Journal de l'école polyt. cah. 19 p. 382 dessen Unrichtigkeit zu erweisen gesucht und dasselbe später in den Mém. de l'acad. des sc. T. VIII. J. 367 und ausfülirlicher T. IX. p. 89 wiederholt. Fourier hat hierauf zuerst kurz in T. VIII. p. 616 und ausführlicher T. X. p. 119 geantworlet. 
noch 6 Null sind. Dagegen lärst sie es unbestimmt, ob die gegebene Gleichung nicht $W$ urzeln habe, die blofs aus einem imaginären Theile bestehen, also in der Form $b \sqrt{ }-1$ enthalten sind. Ich habe früher (\$. 29.) gezeigt, dafs die Gleichung

$$
\text { A. }(4-3 \mu l) \sin \mu l-4 \mu l \cos \mu l=0
$$

keine imaginären $W$ urzeln hat: weder solche, bei welchen der reelle Theil Null ist, noch andere, welche aus einem reellen und einem imaginären Theile bestehen *). Auf diese Gleichung wird Poisson in seinen Untersuchungen über die Schwingungen einer elastischen Kugel geführt**;), und er zeigt auf folgende Weise, dals sie keine imaginären Wurzeln hat. Er findet nemlich durch dieselbe Untersuchung auch noch die Gleichung

$$
\text { B. }\left(\mu^{2}-\mu^{\prime 2}\right) \int_{0}^{l} \boldsymbol{R} R^{\prime} \partial \boldsymbol{r}=0,
$$

wo $\mu$ und $\mu^{\prime}$ zwei Wurzeln der Gleichung (A.) bedeuten, die so beschaffen sind, dafs $\mu^{2}$ und $\mu^{\prime 2}$ verschiedene Werthe haben, also $\mu^{2}-\mu^{\prime 2}$ nicht Null und $R=(\mu r \cos \mu r-\sin \mu r) \frac{1}{r}$ ist, $R^{\prime}$ dagegen Dasjenige bedeutet, was $\boldsymbol{R}$ wird, wenn man in dem Werthe von $R$ statt $\mu$ den Werth $\mu^{\prime}$ substituirt. Hätte nun die Gleichung (A.) imaginäre Wurzeln, so dals etwa $p+q \sqrt{ }-1$ and $p-q \sqrt{ }-1$ ein Paar solcher Wurzeln wären, so könnte man $\mu=$ $p+q \checkmark-1$ and $\mu^{\prime}=p-q \checkmark-1$ setzen and durch $\boldsymbol{R}=\boldsymbol{P}+\boldsymbol{Q} r-1$ und $\boldsymbol{R}^{\prime}=\boldsymbol{P}-\boldsymbol{Q} \checkmark-1$ die entsprechenden Werthe von $\boldsymbol{R}$ und $\boldsymbol{R}^{\prime}$ bezeichnen. Die Gleichung $(\boldsymbol{B}$.$) ginge also in$

$$
\int_{0}^{l}\left(\boldsymbol{P}^{2}+\boldsymbol{Q}^{2}\right) \partial \boldsymbol{r}=0
$$

über. Da aber alle Elemente dieses Integrals positiv sind, so könnte es nicht Null werden, wenn man nicht $\boldsymbol{P}=0$ und $\boldsymbol{Q}=0$ für jeden Werth von $\boldsymbol{r}$ hätte. Aus diesen Werthen würden sich also Werthe von $p$ und $q$ ergeben, die von $\boldsymbol{r}$ abhängen; was unzuläfslich ist: also kann auch die Voraussetzung, dafs die Gleichung imaginäre Wurzeln habe, nicht richtig sein. Indessen sieht man, dafs dieser Schlufs darauf beruht, dafs $\mu^{2}$ und $\mu^{\prime 2}$ ungleiche Werthe haben. Hätte aber die Gleichung (A.) zwei Wurzeln $q \checkmark-1$, $-q \vee-1$ und man substituirte dieselben statt $\mu$ und $\mu^{\prime}$, so würde der Ausdruck (B.) Null werden, ohne dafs $\int R R^{\prime} \partial r$ Null wird, und also der Schlufs nicht mehr gelten * * * *

$\left.{ }^{*}\right)$ Man erhält nemlich diese Gleichung, wenn man in der dort behandelten Gleichung $(4-3 x) \sin x-4 x \cos x=0$ statt $x$ den Werth $\mu l$ substituirt.

$\left.{ }_{* *}\right)$ Mémoire de l'acad. des sc. T. VIII. p. 417.

***) Eine andere Beweisführung von Sturm findet sich auch im Journ. des Mathém. par Liouville Nov. 1836 p. 384. 
33. Wir wollen nun zeigen, in wiefern die Bernonllische Methode auch auf die transcendenten Gleichungen angewendet werden darf. (Vergl. 5. 1.) Diese Methode beruht wesentlich auf der Eigenschaft der algebraischen Gleichungen, dafs der erste Theil derselben das Product einer Anzahl einfacher, reeller oder imaginärer Factoren vom ersten Grade ist. Es folgt nemlich aus dieser Eigenschaft, dals die Coefficienten der einzelnen Glieder der Gleichung bestimmte Functionen der Wurzeln sind; und gerade diese Eigenschaft ist es, auf welcher die Anwendung der recurrirenden Reihen auf die Auflösung der Gleichungen beruht. Es ergiebt sich mithin von selbst, dafs die recurrirenden Reihen keinesweges zur allgemeinen Auflösung der transcendenten Gleichungen angewendet werden können. Denn es ist im Früheren nachgewiesen worden, dafs der erste Theil einer transcendenten Gleichung häufig von dem Producte der den Wurzeln der Gleichung entsprechenden Factoren wesentlich verschieden ist (\$. 5. u. 6.). Auf eine solche Gleichung läfst sich also die Bernoullische Methode nicht anwenden, weil ihr diejenige Eigenschaft fehlt, auf welcher allein sie gegründet ist; oder man muls wenigstens im Stande sein, den Factor des ersten Theils der Gleichung zu finden, welcher dem Producte aller den Wurzeln der Gleichung entsprechenden Factoren gleich ist. Sobald indessen die transcendente Gleichung von der Art ist, dafs ihr erstes Glied das Product von Factoren ist, die den Wurzeln der Gleichung entsprechen, so werden auch die Coefficienten der einzelnen Glieder der Gleichung auf dieselbe Weise, wie es bei den algebraischen Gleichungen der Fall ist, aus den Wurzeln der Gleichung zusammengesetzt sein, und es kann daher bei solchen transcendenten Gleichungen die Bernoullische Methode auf dieselbe Weise wie bei den algebraischen gebraucht werden.

Es ist schon oben (\$. 1.) bemerkt worden, dafs die Ausbildung der Bernoullischen Methode früher sehr mangelhaft war. Gegenwärtig besitzt man mehrere Bearbeitungen derselben, welche zeigen, wie man alle Wurzeln algebraischer Gleichungen finden kaun und die daher obne Schwierigkeit auf solche transcendente Gleichungen angewandt werden können, welche der oben angegebenen Bedingung entsprechen.

Ich habe zuerst *) mit Hülfe der bei Fourier vorkommenden Andeutungen eine solche Methode nachgewiesen. Einfacher ist die Methode,

*) Crelle's Journ. für die Mathem. Bd. 11. p. 293 ff. 
welche Jacobi später gegeben hat *) und die ohne Zweifel mit derjenigen, welche Fourier selbst beabsichtigte, ganz identisch ist.

Ich werde mich hier begnügen, zu zeigen, wie man vermittelst dieser letzteren Methode die zwei grölsten oder kleinsten, reellen oder imaginären Wurzeln der transcendenten Gleichungen finden kann. In Beziehung auf algebraische Gleichungen habe ich das hier anzuwendende Verfahren bereits früher mitgetheilt ${ }^{*}$ ).

34. Es sei also eine transcendente Gleichung $F x=\left(1-\frac{x}{\alpha}\right)\left(1-\frac{x}{\beta}\right)\left(1-\frac{x}{\gamma}\right)\left(1-\frac{x}{\delta}\right) \ldots=1-\boldsymbol{A} x+B x^{2}-C x^{3}+D x^{4} \ldots$ gegeben, so dafs $\alpha, \beta, \gamma, \delta \ldots$ die reellen oder imaginären Wurzeln der Gleichung sind; nach der zunehmenden Gröfse geordnet. Setzt man

$$
\begin{aligned}
& A_{1}=\frac{1}{\alpha}+\frac{1}{\beta}+\frac{1}{\gamma}+\frac{1}{\delta}+\ldots \\
& A_{2}=\frac{1}{\alpha^{2}}+\frac{1}{\beta^{2}}+\frac{1}{\gamma^{2}}+\frac{1}{\delta^{2}}+\ldots \\
& A_{3}=\frac{1}{\alpha^{3}}+\frac{1}{\beta^{3}}+\frac{1}{\gamma^{3}}+\frac{1}{\delta^{3}}+\ldots \\
& \ldots \ldots \\
& A_{n}=\frac{1}{\alpha^{n}}+\frac{1}{\beta^{n}}+\frac{1}{\gamma^{n}}+\frac{1}{\delta^{n}}+\ldots
\end{aligned}
$$

so hat man bekanntlich

$$
\begin{aligned}
& \boldsymbol{A}_{1}=\boldsymbol{A} \\
& \boldsymbol{A}_{2}=\boldsymbol{A} \boldsymbol{A}_{1}-2 \boldsymbol{B} \\
& \boldsymbol{A}_{3}=\boldsymbol{A} \boldsymbol{A}_{2}-B \boldsymbol{A}_{1}+3 \boldsymbol{C}
\end{aligned}
$$

Ist nun a die kleinste Wurzel und zugleich reell, so kann man, wenn $n$ grofs genug genommen wird, näherungsweise

$$
\begin{aligned}
A_{n} & =\frac{1}{a^{n}}, \\
A_{n+1} & =\frac{1}{a^{n+1}},
\end{aligned}
$$

setzen; und hieraus ergiebt sich der Werth der kleinsten Wurzel $\alpha=\frac{A_{n}}{A_{n+1}}$.

Bildet man daher eine Reihe, deren allgemeines Glied $\boldsymbol{A}_{n}$ ist, und dividirt jedes Glied dieser Reihe durch das folgende, so nähert sich der

*) Crelle's Journ. für die Mathem. Bd. 13. p. 399 ff.

${ }^{* *}$ Ebend. Bd. 9. p. 305. 
Quotient immer mehr dem Werthe $a$. Diese Reihe werde ich im Folgenden $(\boldsymbol{S})$ nennen.

Setzt man ferner näherungsweise

$$
\begin{aligned}
A_{n} & =\frac{1}{\alpha^{n}}+\frac{1}{\beta^{n}} \\
A_{n+1} & =\frac{1}{\alpha^{n+1}}+\frac{1}{\beta^{n+1}}, \\
A_{n+2} & =\frac{1}{\alpha^{n+2}}+\frac{1}{\beta^{n+2}}, \\
A_{n+3} & =\frac{1}{\alpha^{n+3}}+\frac{1}{\beta^{n+3}}, \\
A_{n+4} & =\frac{1}{\alpha^{n+4}}+\frac{1}{\beta^{n+4}},
\end{aligned}
$$

so findet man

$$
\begin{gathered}
A_{n} \cdot A_{n+2}-\left(A_{n+1}\right)^{2}=\frac{1}{\alpha^{n}} \cdot \frac{1}{\beta^{n}}\left(\frac{1}{\alpha}-\frac{1}{\beta}\right)^{2} \\
A_{n+1} \cdot A_{n+3}-\left(A_{n+2}\right)^{2}=\frac{1}{\alpha^{n+1}} \cdot \frac{1}{\beta^{n+1}}\left(\frac{1}{\alpha}-\frac{1}{\beta}\right)^{2}, \\
A_{n+2} \cdot A_{n+4}-\left(A_{n+3}\right)^{2}=\frac{1}{\alpha^{n+2}} \cdot \frac{1}{\beta^{n+2}}\left(\frac{1}{\alpha}-\frac{1}{\beta}\right)^{2},
\end{gathered}
$$

also

$$
\frac{A_{n} \cdot A_{n+2}-\left(A_{n+1}\right)^{2}}{A_{n+1} \cdot A_{n+3}-\left(A_{n+2}\right)^{2}}=\alpha \beta \text {. }
$$

Bildet man daher eine Reihe, deren allgemeines Glied $\boldsymbol{A}_{n} \boldsymbol{A}_{n+2}-\left(\boldsymbol{A}_{n+1}\right)^{2}$ ist, und dividirt jedes Glied durch das folgende, so nähert man sich immer mehr dem Werthe $\alpha \beta$. Diese Reihe werde ich im Folgenden $\left(\boldsymbol{S}_{1}\right)$ nenuen.

Ist $a$ reell, und hat man seinen Werth aus der Reihe $\left(\boldsymbol{S}_{1}\right)$ gefunden, so findet man den Werth von $\beta$, wenn man den aus der Reihe $\left(S_{1}\right)$ gefundenen Werth von $\alpha \beta$ durch den gefundenen Werth von $\alpha$ dividirt. Ist dagegen a imaginär, so divergirt die Reihe $\left(\boldsymbol{S}_{1}\right)$ und es kann der Werth von $\alpha$ nicht aus derselben gefunden werden. Die Reihe $\left(\boldsymbol{S}_{\mathbf{1}}\right)$ convergirt aber auch in diesem Falle und giebt den Werth von $\alpha \beta$. Um nan auch in diesem Falle die Werthe von $\alpha$ und $\beta$ zu finden, bilde man eine dritte Reihe $\left(\boldsymbol{S}_{2}\right)$, deren allgemeines Glied

$$
\frac{A_{n} \cdot A_{n+3}-A_{n+1} \cdot A_{n+2}}{A_{n} A_{n+2}-\left(A_{n+1}\right)^{2}}
$$

ist. Die Glieder dieser Reihe nähern sich dem Werthe $\frac{1}{\alpha}+\frac{1}{\beta}$ desto melır, 
je gröfser $n$ ist; und diese Reihe wird immer convergiren, da $\alpha$ und $\beta$ ein zusammengehörendes Paar imaginärer Wurzeln sind. Aus $\alpha \beta$ und $\frac{1}{\alpha}+\frac{1}{\beta}$ findet man alsdann den Werth von $\alpha$ und $\beta$.

35. Ich will diese Erörterungen zunächst anwenden, um die zwei kleinsten Wurzeln der Gleichung

$$
1-x+\frac{x^{2}}{2^{2}}-\frac{x^{3}}{2^{2} \cdot 3^{2}}+\frac{x^{4}}{2^{2} \cdot 3^{3} \cdot 4^{2}}-\ldots=0
$$

zu finden. Bildet man die Reihe $(\boldsymbol{S})$, so ergiebt sich

$$
1, \frac{1}{2}, \frac{1}{3}, \frac{33}{144}, \frac{19}{120}, \frac{473}{4320}, \frac{229}{3024}, \frac{101369}{1935360}, \ldots
$$

Dividirt man das letzte Glied durch das vorletzte, so findet man als Näherungswerth der kleinsten Wurzel 1,4458....; was mit dem früher gefundenen Werthe (\$. 27.) übereinstimmt.

Die ersten Glieder der Reihe $\left(\boldsymbol{S}_{1}\right)$ sind

$\frac{1}{12}, \frac{1}{288}, \frac{1}{3840}, \frac{23}{1036800}, \frac{257}{130636800}, \frac{10359}{58525286400}, \ldots$

Dividirt man das vorletzte Glied durch das letzte, so findet man 11,1145 .... welches also das Product der zwei kleinsten Wurzeln ist, und wenn man diesen Werth durch den gefundenen Werth der ersten Wurzel dividirt, so ergiebt sich als Näherungswerth der zweiten Wurzel 7,68 ...; was schon bis zur zweiten Decimalstelle mit dem oben gefundenen Werthe übereinstimmt.

36. Ich will bei dieser Gelegenheit einen Lehrsatz beweisen, den Fourier in dem Exposé synoptique *) angedeutet hat. Fourier sagt nemlich, dafs wenn man vermittelst der angegebenen Methode die erste Wurzel sucht, und diese reell ist, dafs alsdann die Reihe der Quotienten so gegen den wahren Werth der Wurzel convergire, dafs zuletzt die Fehler, welche man bei der Annäherung begeht, wie die Glieder einer geometrischen Progression abnehmen, deren Exponent das Verhältnifs der zweiten Wurzel zur ersten sei. Hierbei wird vorausgesetzt, dafs die erste Wurzel die grörste ist. In dem anderen Falle, welcher uns hier besonders interessirt, wo nemlich die erste Wurzel die kleinste ist, mufs dieser Satz so modificirt werden, dafs man als Exponenten der geometrischen Progression das Verhältnifs der ersten Wurzel zur zweiten nehmen muls. Der Beweis ist

*) Analyse des équations p. 71 .

Crelle's Journal d. M. Bd. XXIL. Hfh 1. 
für beide Fälle derselbe; ich werde daher nur den zweiten berücksichtigen. Um den Werth der Wurzel $\alpha$ zu finden, setzt man $\frac{A_{n}}{A_{n+1}}=\alpha$ : in Wahrheit ist aber

$$
\frac{A_{n}}{A_{n+1}}=\alpha+\frac{\frac{1}{\beta^{n}}+\frac{1}{\gamma^{n}}+\frac{1}{\delta^{n}}+\ldots .-\alpha\left(\frac{1}{\beta^{n+1}}+\frac{1}{\gamma^{n+1}}+\frac{1}{\delta^{n+1}}+\ldots .\right)}{\frac{1}{a^{n+1}}+\frac{1}{\beta^{n+1}}+\frac{1}{\gamma^{n+1}}+\frac{1}{\delta^{n+1}}+\ldots} .
$$

Der begangene Fehler ist also

$$
\frac{\frac{1}{\beta^{n}}+\frac{1}{\gamma^{n}}+\frac{1}{\delta^{n}}+\ldots-\alpha\left(\frac{1}{\beta^{n+1}}+\frac{1}{\gamma^{n+1}}+\frac{1}{\delta^{n+1}}+\ldots\right)}{\frac{1}{\alpha^{n+1}}+\frac{1}{\beta^{n+1}}+\frac{1}{\gamma^{n+1}}+\frac{1}{\delta^{n+1}}+\ldots} .
$$

Ist jedoch $n$ hinlänglich grofs, so kann man statt dessen auch

$$
\frac{\frac{1}{\beta^{n}}-\alpha \cdot \frac{1}{\beta^{n+1}}}{\frac{1}{\alpha^{n+1}}+\frac{1}{\beta^{n+1}}}=\frac{(\beta-\alpha)}{1+\frac{\alpha^{n+1}}{\beta^{n+1}}} \cdot \frac{\alpha^{n+1}}{\beta^{n+1}}
$$

setzen, und da, wenn $n$ sehr grofs, $\frac{a^{n+1}}{\beta^{n+1}}$ ein so kleiner Bruch ist, dafs man ihn gegen die Einheit vernachlässigen kann, so convergiren die Fehler gegen die Grenze $(\beta-\alpha) \cdot\left(\frac{\alpha}{\beta}\right)^{n+1}$ hin und nehmen mithin im Verbältnifs der Glieder einer geometrischen Progression ab, deren Exponent $\frac{\alpha}{\beta}$ ist.

37. Um auch noch ein Beispiel der Berechnung der zwei kleinsten imaginären Wurzeln zu geben, nehme ich die Gleichung

$$
x=e^{x} \text {. }
$$

Diese Gleichung kann keine reelle Wurzel haben, da, so lange $x$ reell ist, - immer $x<e^{x}$ ist. Entwickelt man $e^{x}$ in eine Reihe, so gelt die Gleichung in folgende über:

oder in

$$
x=1+x+\frac{x^{2}}{2}+\frac{x^{3}}{2.3}+\frac{x^{4}}{2.3 .4}+\cdots
$$

$$
1+\frac{x^{2}}{2}+\frac{x^{3}}{2.3}+\frac{x^{4}}{2.3 .4}+\ldots=0 \text {. }
$$

Vergleicht man diese Gleichung mit der algebraischen Gleichung

$$
1+\frac{n \cdot n-1}{2} y^{2}+\frac{n \cdot n-1 \cdot n-2}{1 \cdot 2 \cdot 3} y^{3}+\ldots=0,
$$

so kann man wieder zeigen, dafs sie das Product einer unendlichen Anzahl von Factoren ist. Sie kann millin durch die Bernoullische Methode aufgelöset werden. 
1. Stern, über die Auflösung der transcendenten Gleichungen.

Bildet man die Reihe $(S)$, so ergiebt sich
$\begin{array}{llllllll}\boldsymbol{A}_{1} & \boldsymbol{A}_{2} & \boldsymbol{A}_{3} & \boldsymbol{A}_{\mathbf{6}} & \boldsymbol{A}_{5} & \boldsymbol{A}_{6} & \boldsymbol{A}_{7}\end{array}$
$\boldsymbol{A}_{8}$
$\boldsymbol{A}_{9}$
$0-1-\frac{1}{2}+\frac{1}{3}+\frac{3}{8} \frac{-1}{4.5} \frac{-31}{2.3 .4 .6} \quad \frac{-29}{3.5 .6 .7} \quad \frac{+63}{2^{2} .4 .5 .8} \frac{+2087}{3.4 .6 .7 .8 .9} \cdots$
$0-1-\frac{1}{2}+\frac{1}{3}+\frac{3}{8} \frac{-1}{4.5} \frac{-31}{2.3 .4 .6} \quad \frac{-29}{3.5 .6 .7} \quad \frac{+63}{2^{2} .4 .5 .8} \frac{+2087}{3.4 .6 .7 .8 .9} \cdots$
$A_{11}$

Hieraus ergiebt sich

$$
\begin{aligned}
\frac{A_{7} A_{9}-A_{8}^{2}}{A_{8} A_{10}-A_{9}^{2}} & =\frac{1364243616}{722045476}=1,889415 \text { und } \\
\frac{A_{7} A_{10}-A_{8} A_{9}}{A_{7} A_{9}-A_{8}^{2}} & =\frac{14356678}{42} \frac{632613}{6326}=0,336753 .
\end{aligned}
$$

Nennt man nun die kleinsten Wurzeln $\alpha$ und $\beta$, so ist

$$
\begin{aligned}
\alpha \beta & =1,889415 \text { und } \\
\frac{\alpha+\beta}{\alpha \beta}=\frac{1}{\alpha}+\frac{1}{\beta} & =0,336753 ;
\end{aligned}
$$

mithin kann man, da $\alpha$ und $\beta$ ein Paar conjugirte imaginäre Wurzeln sind,

$$
a=a+b r-1 \text { und } \beta=a-b \sqrt{ }-1
$$

setzen und findet vermiltelst der Werthe von $\alpha \beta$ und $\frac{\alpha+\beta}{\alpha \beta}$ :

$$
\begin{aligned}
& \boldsymbol{a}=0,318133, \\
& \boldsymbol{b}=1,337238 .
\end{aligned}
$$

Die Wurzeln sind also $0,318133 \pm 1,337238 \checkmark-1$.

Cauchy ${ }^{*}$ ) findet auf anderem Wege

$$
0,3181317 \pm 1,337235 \% \checkmark-1 \text {. }
$$

38. Während ich im Begriff bin diese Abhandlung zu endigen, erhalte ich das Compte rendu No. 10. 1837, welchesseine neue Methode von Cauchy enthält, die Näherungswerthe der reellen Wurzeln zu finden, welche zugleich auf transcendente Gleichungen anwendbar ist. Das Wesentlichsie derselben ist in Folgendem enthalten. Es sei

$$
\text { 1. } f x=0
$$

eine Gleichung, deren erstes Glied $f x$ zwischen den Grenzen $x=x_{0}$ und $x=\boldsymbol{X}$ continuirlich bleibt. Ferner sei die Function $f x$ in zwei andere

$$
\varphi x-\chi x
$$

zerlegt, die ebenfalls zwischen den gegebenen Grenzen continuirlich und so beschaffen sind, dals die Werthe von $\varphi_{x}$ und' $\chi x$ mit den Werthen von $x$ wachsen. Nennt man nun $-\frac{1}{\alpha}$ das kleinste und $-\frac{1}{\beta}$ das gröfste der Verhältnisse

$$
\frac{\varphi^{\prime} x^{0}-\chi^{\prime} X}{f x_{0}}, \quad \frac{\varphi^{\prime} X-\chi^{\prime} x_{0}}{f x_{0}}
$$

*) Leçons sur le calc. différ., leç. 14. 
und $\frac{1}{A}$ das kleinste, $\frac{1}{B}$ das gröfste der Verhältnisso

$$
\frac{\varphi^{\prime} x_{0}-\chi^{\prime} X}{f X}, \quad \frac{\varphi^{\prime} X-\chi x_{0}}{f X},
$$

und sind zwischen $x_{0}$ und $\boldsymbol{X}$ Wurzeln der Gleichung enthalten, so sind auch $x_{0}+\alpha, \boldsymbol{X}-\boldsymbol{A}$ zwischen diesen Grenzen enthalten und bilden zugleich Grenzen jener Wurzeln. Ist übrigens eine der Grölsen $x_{0}+\beta, \boldsymbol{X}-\boldsymbol{B}$, zwischen den Grenzen $x_{0}$ und $\boldsymbol{X}$ enthalten, so hat die Gleichung (1.) gewirs Wurzeln zwischen diesen Grenzen.

Ist $f x$ eine ganze Function, so kann man sie immer leicht in $\varphi x-\chi x$ zerlegen, wenn man für $x_{0}$ und $\boldsymbol{X}$ positive Grenzen nimmt, illdem man die Summe der positiven Glieder durch $\varphi_{x}$, die Summe der negativen Glieder durch $-\chi x$ bezeichnet. Da man aber die negativen Wurzeln einer Gleichung, wie bekannt, immer in positive verwandeln kann, wenn man $-x$ statt $x$ setzt, so kann man auch durch dieses Verfahren alle Wurzeln einer algebraischen Gleichung mit jedem beliebigen Grade von Genauigkeit finden, sobald man zwei Werthe kennt, zwischen welchen die Wurzeln enthalten sind. Dasselbe findet bei einer transcendenten Gleichung

$$
f x=0
$$

stalt, sobald man die Function $f x$ in zwei andere $\varphi_{x}-\chi x$ zerlegen kann, die so beschaffen sind, dafs $\varphi x$ und $\chi x$ immer wachsen, wenn $x$ grölser wird.

Ich will nun zeigen, wie dieses Verfahren auch aus Fourier's Methode abgeleitet werden kann.

Ist nemlich eine Gleichung $f x=0$ gegeben, und ist eine Wurzel zwischen zwei Grenzen enthalten, so kann man immer so enge Grenzen $\boldsymbol{x}_{0}$ und $\boldsymbol{X}$ ziehen, dafs eines der vier folgenden Schemata Statt findet:

$$
\begin{aligned}
& \text { I. }\left\{\begin{array}{l}
{\left[x_{1}\right]=\ldots+x+f^{\prime \prime}+f x} \\
{[X]=\ldots+t+}
\end{array}\right.
\end{aligned}
$$

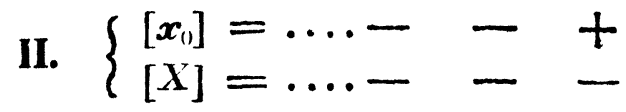

$$
\begin{aligned}
& \text { III. }\left\{\begin{array}{l}
{\left[x_{0}\right]=\ldots+-t} \\
{[X]=\ldots+-t}
\end{array}\right. \\
& \text { IV. }\left\{\begin{array}{l}
{\left[\boldsymbol{x}_{0}\right]=\ldots++{ }^{+}+} \\
{[\boldsymbol{X}]=\ldots+}
\end{array}\right.
\end{aligned}
$$


In den zwei ersten Fällen hat man die Näherungswerthe

$$
X-\frac{f X}{f^{\prime} X}>x, \quad x_{0}-\frac{f x_{0}}{f^{\prime} X}<x,
$$

in den zwei andern die Näherungswerthe

$$
X-\frac{f X}{f^{\prime} x_{0}}>x, \quad x_{0}-\frac{f x_{0}}{f^{\prime} x_{0}}<x .
$$

Setzt man nun $f x=\phi_{x}-\chi x$, also $f^{\prime} x=\phi^{\prime} x-\chi^{\prime} x$, so gehen diese Werthe in folgende über. Nemlich in den zwei ersten Fällen hat man

$$
\boldsymbol{X}-\frac{f X}{\varphi^{\prime} X-\chi^{\prime} \bar{X}}>x, \quad x_{0}-\frac{f x_{0}}{\varphi^{\prime} X-\chi^{\prime} X}<x
$$

und in den zwei anderen

$$
\boldsymbol{X}-\frac{f \boldsymbol{X}}{\varphi^{\prime} x_{0}-\chi^{\prime} x_{0}}>x, \quad x_{0}-\frac{f x_{0}}{\varphi^{\prime} x_{0}-\chi^{\prime} x_{0}}<x .
$$

Im ersten Falle ist $\phi^{\prime} X-\chi^{\prime} X$ positiv und kleiner als $\varphi^{\prime} X-\chi^{\prime} x_{0}$, mithin ist auch der letztere Ausdruck positiv, folglich, da $f \boldsymbol{X}$ positiv ist,

mithin

$$
\frac{f X}{\varphi^{\prime} X-\chi^{\prime} x_{0}}<\frac{f X}{\varphi^{\prime} X-\chi^{\prime} X}
$$

$$
\boldsymbol{X}-\frac{f X}{\varphi^{\prime} X-\chi^{\prime} x_{0}}>\boldsymbol{X}-\frac{f X}{\varphi^{\prime} X-\chi^{\prime} X}>x .
$$

Nun ist $\varphi^{\prime} X-\chi^{\prime} x_{0}>\varphi^{\prime} x_{0}-\chi^{\prime} X$, also ist, nach Cauchy's Bezeichnung,

und $\boldsymbol{X}-\boldsymbol{A} \leq \boldsymbol{x}$

$$
\frac{f X}{\varphi^{\prime} X-\chi^{\prime} x^{0}}=A
$$

Eben so findet man im vierten Falle, dafs $\varphi^{\prime} x_{0}-\chi^{\prime} x_{0}$ positiv und kleiner als $\varphi^{\prime} \boldsymbol{X}-\chi^{\prime} x_{0}$ ist; also mufs auch letzterer Ausdruck positiv sein. Da nun $f \boldsymbol{X}$ positiv ist, so hat man wieder

$$
X-\frac{f X}{\varphi^{\prime} X-\chi^{\prime} x_{0}}>X-\frac{f X}{\varphi^{\prime} x_{0}-\chi^{\prime} x_{0}}>x,
$$

folglich $X-A \geq \stackrel{x}{X}$.

Im zweiten Falle dagegen ist $-\left(\Phi^{\prime} X-\chi^{\prime} X\right)$ positiv, oder $\chi^{\prime} X-\varphi^{\prime} X$ positiv. Nun ist $\chi^{\prime} \boldsymbol{X}-\varphi^{\prime} x_{0}>\chi^{\prime} \boldsymbol{X}-\varphi^{\prime} \boldsymbol{X}$, folglich auch $-\left(\varphi^{\prime} x_{0}-\chi^{\prime} \boldsymbol{X}\right)$ positiv, und da $f \boldsymbol{X}$ negativ ist,

$$
X-\frac{f X}{\varphi^{\prime} x_{0}-\gamma^{\prime} \bar{X}}>X-\frac{f X}{\varphi^{\prime} X-\chi^{\prime} X}>X
$$


Offenbar ist aber hier $\frac{\varphi^{\prime} x_{0}-\chi^{\prime} X}{f X}=\frac{\chi^{\prime} X-\varphi^{\prime} x_{0}}{-f X}$ gröfser als $\frac{\varphi^{\prime} X-\chi^{\prime} x_{0}}{f X}=\frac{\chi^{\prime} x_{0}-\varphi^{\prime} X}{-f X}$, mithin nach Cauchy's Bezeichnung $\frac{f X}{\varphi^{\prime} x_{0}-\chi^{\prime} X}=A$, und daher $X-1 \geq x$ Auf dieselbe Weise kann man zeigen, dafs auch im dritten Falle

$$
\boldsymbol{X}-\frac{\boldsymbol{f} \boldsymbol{X}}{\varphi^{\prime} x_{0}-\chi^{\prime} \boldsymbol{X}}>\boldsymbol{X}-\frac{\boldsymbol{f} \boldsymbol{X}}{\varphi^{\prime} x_{0}-\chi^{\prime} x_{0}}>\boldsymbol{X} \text { ist. }
$$

Dieselbe Betrachtung führt nun auch zur Bestimmung des zweiten Näherungswerthes, der zwischen $x_{0}$ und $x$ liegt.

Im ersten Falle ist $\varphi^{\prime} x-\chi^{\prime} X$ positiv, also auch der gröfsere Werth $\varphi^{\prime} \boldsymbol{X}-\chi^{\prime} x_{0}$ positiv. Da nun $f x_{0}$ negativ ist, so ist $\frac{-f x_{0}}{\varphi^{\prime} X-\chi^{\prime} x_{0}}$ etwas kleineres Positives als $\frac{-f x_{0}}{\varphi^{\prime} X-\chi^{\prime} \bar{X}}$, mithin

$$
x_{0}-\frac{f x_{0}}{\varphi^{\prime} \boldsymbol{X}-\chi^{\prime} x_{0}}<x_{0}-\frac{f x_{0}}{\varphi^{\prime} X-\chi^{\prime} \boldsymbol{X}}>x_{0},
$$

und da $\varphi^{\prime} X-\chi^{\prime} x_{0}>\varphi^{\prime} x_{0}-\chi^{\prime} X$, so ist nach Cauchy's Bezeichung

Eben so findet man im vierten Falle

$$
\begin{gathered}
x_{0}-\frac{f x_{0}}{\varphi^{\prime} X-\chi^{\prime} x_{0}}=x_{0}+\alpha \text {, indem, da } f x_{0} \text { negativ ist, } \\
\frac{\varphi^{\prime} X-\chi^{\prime} x_{0}}{f x_{0}}<\frac{\varphi^{\prime} x_{0}-\chi^{\prime} X}{f x_{0}} \text { ist. }
\end{gathered}
$$

$$
x_{0}-\frac{f x_{0}}{\varphi^{\prime} \boldsymbol{X}-\chi^{\prime} x_{0}}<x_{0}-\frac{f x_{0}}{\varphi^{\prime} x_{0}-\chi^{\prime} x_{0}}>x_{0} .
$$

Im zweiten Falle findet man

und im dritten

$$
x_{0}-\frac{f x_{0}}{\varphi^{\prime} x_{0}-\chi^{\prime} X}<x_{0}-\frac{f x_{0}}{\varphi^{\prime} X-\chi^{\prime} X}>x_{0}
$$

$$
x_{0}-\frac{f x_{0}}{\varphi^{\prime} x_{0}-\chi^{\prime} X}<x_{0}-\frac{f x_{0}}{\varphi^{\prime} x_{0}-\chi^{\prime} x_{0}}>x_{0} .
$$

Es ergiebt sich aus dieser Uebersicht, dals bei der Fourier'schen Methode die Grenzen noch enger sind als bei der von Cauchy. 